\title{
LA INTEGRIDAD DEL REGISTRO DE DEFUNCIONES ADULTAS EN MÉXICO: 1930-1980
}

\author{
Alejandro Mina Valdés \\ El Colegio de México
}

EN UNA SESIÓN INFORMAL sobre demografía matemática en la reunión de la Asociación Internacional para el Estudio Científico de la Población, llevada a cabo en Liège en 1973, el profesor W. Brass propuso un método para estimar la integridad con la cual las defunciones son registradas (en una encuesta o registro) en una población que es aproximadamente estable. El método está basado en la constancia de la tasa de crecimiento de diferentes segmentos de poblaciones estables. Graficando la tasa de mortaliclad por encima de la edad $a$ contra la razón $N(a) / N(a+)$, donde $N(a)$ es el número de personas de edad $a$ (estimado como el promedio del número de personas alrededor de la edad $a$ ) y $N(a+)$ es el número de personas de edad $a$ o más. La población por encima de la edad $a$ puede ser considerada una población estable: llegar a la edad $a$ es equivalente a nacer dentro de la población en edad $a$ y más. El número de defunciones arriba de la edad $a[D(a+)]$ dividido por $N(a+)$ es la tasa de mortalidad de la población arriba de la edad $a ; N(a) / N(a+)$ es la tasa de "natalidad" si las personas que anualmente alcanzan la edad $a$ son consideradas nacidas dentro de la población de personas de edad $a$ y por encima. La tasa de natalidad menos la tasa de mortalidad es la tasa de crecimiento; en una población estable la tasa de crecimiento de la población arriba de la edad $5,10, \ldots, 70$ es la misma. Asi, $D(a+) / N(a+)$ y $\dot{N}(a) / N(a+)$ en las distintas edades difieren por una constante $[r(a+)=r]$. Cuando $D(a+) / N(a+)$, los puntos resultantes deben describir una línea recta con una pendiente de uno, donde las dos variables difieren por una constante, y son entonces equivalentes a la ecuación de una línea recta: $Y=x+r$. Si solamente es registrada una fracción $K$ de defunciones en cada edad, la pendiente de la línea es $K$ veces más grande que la unidad. De aquí que una gráfica de $D(a+) / N(a+)$ contra $N(a) / N(a+)$ provee una serie de puntos para los cuales una línea recta puede ser ajustada; la pendiente de la linea es la integridad del registro de defunciones relativo a la integridad del registro de la población. A pesar de que el procedimiento de Brass está basado en la constancia de la tasa de crecimiento de los diferentes segmentos de una población estable, no requiere, a priori, conocer la tasa de crecimiento; en realidad, del método mismo se deriva una estimación de la tasa de crecimiento

Una descripción completa se encuentra en Brass (1975). 
En 1978, Preston (Preston y Hill, 1980) proponen un segundo procedimiento para determinar la integridad con que son registradas las defunciones en poblaciones aproximadas estables. Cabe señalar que ambos procedimientos (Brass y Preston) son convencionalmente limitados por la mortalidad infantil, esto es, para la mortalidad por debajo de 5010 años. La integridad del registro de defunciones (del sistema de registro o de registro de una encuesta) para infantes y niños es a menudo diferente de la integridad del registro de defunciones de edades adultas. Cualquier acontecimiento de la estimación de la mortalidad infantil y en los primeros años de vida puede ser tratado separadamente por métodos que actualmente están bien establecidos (Brass y Coale, 1968; Sullivan, 1972; Trussell, 1975; Preston y Palloni, 1977).

Uno de los últimos aportes al tema, es el efectuado por Samuel Preston, Ansley J. Coale, James Trussell, y Maxime Weinstein (1980).* En el presente trabajo se presenta el modelo planteado por ellos, así como su empleo en la estimación del grado de subregistro de la mortalidad adulta en México.

El método anteriormente considerado, está basado en características de poblaciones estables, esto es, poblaciones que han estado sujetas por un largo tiempo a pequeñas variaciones en las tasas específicas de mortalidad o en los niveles de fecundidad. Las características esenciales de una población estable son mantenidas si la fecundidad ha cambiado, con tal de que haya tendencias fuertes en la fecundidad, hacia arriba o hacia abajo, existentes 15 o 20 años antes de la fecha en que la población es observada. Recientes cambios en la mortalidad pueden afectar la estructura de la población en edades adultas, pero se debe ver que el efecto sobre la estimación de la integridad del registro de defunciones puede generalmente ser sostenido dentro de ciertos límites de tolerancia.

Antes de mostrar cómo la estimación de la integridad relativa del registro del número de defunciones y personas puede ser derivado del registro de defunciones y personas por edad, nótese que puede ser construida una tabla de vida para una población estable directamente a partir de la distribución del registro de defunciones por edad, o a partir de la distribución por edad de la población.

El punto de partida es la fórmula convencional para obtener el número de personas de edad $x$ en una población estable:

$$
N(x)=B \exp (-r x)[l(x) / l(0)]
$$

donde, para una población dada en un año dado, $N(x)$ es el número de personas de edad $x, B$ es el número de nacimientos, y $r$ es la tasa anual de crecimiento. Usamos la notación de una tabla de vida estándar en todo, excepto que $l x$ ha sido reemplazada por $l(x)$. De (1) se tiene que:

$$
l(x) / l(0)=[N(x) / B] \exp .(r x)
$$

$\mathrm{y}$, de forma más general, que:

$$
l(x) / l(a)=[N(x) / N a] \exp r(x-a)
$$

* Preston, Samuel; Coale, Ansley J.; Trussell, James; ỳ Weinstein, Maxime, 1980. Estimating the completeness of reporting of adult deaths in populations that are aproximately stable. Population Index (Princeton, N.J.) 46 (2): 179-202. 
Para construir una tabla de vida con un radix de 1.0 a la edad 10 de una distribución por edad de personas en una población que se supone estable arriba de la edad 10 , se puede estimar $N(10)$ como una décima parte del número de personas entre las edades exactas 5 y 15, y calcular ${ }_{5} L_{\mathrm{x}}-5 / l(10)$ como. ${ }_{5} N_{\mathrm{x}} / N(10)$ exp $r(x-10+2.5) ; l(x) / l(10)$ es estimada como $\left({ }_{5} L_{x}-5+{ }_{5} L_{x}\right) /[10 \cdot l(10)]$.

El número de defunciones a la edad $x$ en cualquier población es $N(x) \cdot \mu(x)$, donde $\mu(x)$ es la tasa de mortalidad a la edad $x$. Deduciendo de la ecuación (1) que el número de defunciones a la edad $x$ en una población estable es

$$
D(x)=[B / l(x)] \exp (-r x) l(x) \mu(x)
$$

En una población estacionaria con las mismas tasas de mortalidad, y con tasas anuales de natalidad igual al rádix, $1(0)$, la tasa de crecimiento es cero, asi que llega a ser, para dicha población,

$$
d_{0}(x)=l(x) \mu(x)
$$

donde $d_{0}(x)$ es el número de defunciones a la edad $x$ en una población estacionaria. Dividiendo (5) por (4), tenemos que

$$
d_{0}(x) / D(x)=[l(0) / B] \exp (r x)
$$

o que:

$$
d_{0}(x)=C \cdot D(x) \exp (r x)
$$

donde $C$ es una constante. Para construir una tabla de vida con un rádix de 1.0 a la edad 10 a partir de la distribución del número de defunciones por edad en una población estable, se puede hacer uso de la igualdad en una tabla de vida entre $l(a)$ y el número de defunciones $\left[\sum_{\mathrm{x}=a}^{\omega} d_{0}(x)\right]$ arriba de la edad $a$. Así la constante en la ecuación (7) puede ser seleccionada tal que $\stackrel{\omega}{\Sigma} d_{0}(x)=$ j.o; i.e., $C=$ $1 / \sum_{10}^{\omega} D(x) \exp (r x)$. Con esta constante, $l(10)=1.0, l(15)=C \sum_{15}^{\omega} D(x) \exp (r x)$, etc. El cálculo numérico puede estar basado en las defunciones tabuladas en intervalos de edad de 10 a alguna edad avanzada, por ejemplo, $85, y$ las defunciones por encima de los 85 listarlas como un solo número. Entonces $l(a)$ sería calculada de 10 a 80 a través de los pasos sigujentes:

$C=1 / \sum_{10}^{80}\left[{ }_{5} D_{x} \exp [r(x-10+2.5)]+D(85+) \exp [r[75+e(85)]]\right]$

donde $e(85)$ es la esperanza de vida a la edad 85 . Entonces $l(10)=1.0$, y: 
$l(a)=C\left\{\sum_{a}^{80}\left[{ }_{5} D_{x} \exp [r(x-10+2.5)]+D(85+) \exp [r[75+e(85)]]\right]\right\}$

Resumiendo las dos alternativas presentadas anteriormente se tiene que:

1) Una tabla de vida puede ser construida a partir de una población estable vía la multiplicación del número de personas en cada edad por $\exp (r a)$, obteniendo la serie $l(a)$, y el rádix puede ser puesto a cualquier edad conveniente.

2) Una tabla de vida puede ser construida a partir de la distribución de las defunciones, multiplicando el número de defunciones en cada edad por exp( $r a)$, obteniendo la serie $d_{0}(a)$, y $l(a)$ puede entonces ser determinada sumando los valores $d_{0}(x)$ arriba de la edad $a$, con el rádix también puesto a cualquier edad conveniente.

Tasas específicas de mortalidad podrían ser calculadas a partir del número de defunciones registradas y del número de personas en cada intervalo de edad. Supóngase que las defunciones registradas son agrupadas en grupos quinquenales de edad hasta la edad 85 , en un período dado, y en un intervalo abierto para las personas arriba de 85 , y que un censo se levantó a mitad del mismo período listan. do a la población de acuerdo con la misma clasificación por edad. Valores de ${ }_{5} m_{x}$ (para $x=10$ a 80 ) y $m(85+$ ) podrían ser calculados bajo e] supuesto de que en una tabla de vida los valores ${ }_{5} m_{x}$ son iguales a los valores ${ }_{5} m_{x}$ de la pobla. ción; usando la aproximación:

$$
{ }_{5} q_{x}=\frac{5 \cdot\left({ }_{5} m_{x}\right)}{1+(2.5)\left({ }_{5} m_{x}\right)}
$$

y la relación $l(x+5)=l(x)\left(1-{ }_{5} q_{x}\right), l(x)$ para $x=15$ a 85 podría ser determinado, con un rádix $l(10)$ de 1.0 a la edad 10.

La compración de estas tres tablas de vida - una derivada de la tasa de crecimiento y la distribución de la población, otra de la tasa de crecimiento y la distribución de las defunciones, y la tercera de las tasas de mortalidad calculadas a partir del registro del número de defunciones y de personas-podrían servir para revelar al investigador si el supuesto de estabilidad y la exactitud de los datos fundamentales registrados para su construcción son aproximadamente correctos, en cuyo caso todas las tablas coincidirían, o que los supuestos son violados, en cuyo caso ellas diferirían.

En e] modelo descrito en Preston et al. (1980), los autores señalan, después de mostrar la construcción de tablas de vida a partir de las distribuciones por edad antes señaladas, que la función $l(x)$ calculada multiplicando la distribución por edad de la población por erx, conserva los padecimientos y sesgos de la distribución por edad registrada. Debido a la acumulación por edad, a otras formas de registro por edad erróneas, $\mathrm{y}$ a omisiones diferenciales, el registro de la estructura por edad de la población algunas veces posee una tenue semejanza con la verdadera distribución por edad.

La estructura por edad de las curvas $l(x)$ generadas al multiplicar la distribución de las defunciones por $e^{r x}$ y por el cálculo estándar de las tasas específicas 
por edad de mortalidad, son monótonas y relativamente suaves. La función $l(x)$ derivada de las defunciones y la tasa de crecimiento es el resultado de acumular la distribución porcentual estimada de defunciones en una población estacionaria de las edades más avanzadas. Asciende monótonamente trazando la curva hacia atrás desde cerca del cero en las edades mayores al 1.0 a la edad 10. Las defunciones acumuladas deben aumentar cuando disminuye la edad porque el número estimado de defunciones en cada intervalo de edad de la población estacionaria es necesariamente positivo. La tabla de vida basada en la transformación convencional de $m_{x}$ a $q_{x}$ decrece monótonamente de la edad 10 a los valores $l(x)$ más avanzados, donde cualquier $n q_{x}$ se obtiene al restar $l(x+n)$ de $l(x)$.

Los autores muestran los efectos de varios defectos en los datos, o en los supuestos, sobre las tres funciones $l(x)$, los cuales son ilustrados en la figura 1 . Las tres series de tablas de vida fueron calculadas por los autores para una población estable hipotética derivada a partir de una tasa de crecimiento específica y una serie específica de tasas específicas de mortalidad por edad; después la población estable ha sido deliberadamente distorsionada en diferentes formas.* En las gráficas de la figura 1 se observan los siguientes efectos:

1) Subregistro uniforme de defunciones del $20 \%$ (gráfica $A$ ), $l(x)$ calculada a partir de la mortalidad específica por edad es elevada; $l(x)$ de la distribución por edad de las personas o de las defunciones no es afectada.

2) Una estimación incorrecta de la tasa de crecimiento de 0.020 en lugar de una tasa de 0.025 (gráfica B).

3) Violación del supuesto de la integridad constante del registro de defun. ciones por edad, por lo que la integridad aumenta al avanzar la edad (gráfica $\mathrm{C}$ ).

4) Un aumento sobreestimando la edad de las defunciones de las personas viejas a partir de los 55 años (gráfica $D$ ).

5) Un aumento sobreestimando la edad de las personas viejas, empezando a los 55 años (gráfica E).

6) Un aumento sobreestimando la edad de las personas y defunciones de las personas viejas empezando a los 55 años de edad (gráfica F).

7) Una distribución no-estable de la población y de las defunciones, resultado de una población (inicialmente estable, y en todo tiempo con la misma fecundidad específica por edad listada como una población similar) que ha alcanzado una $e(0)$ de 50 años, después un incremento en 25 años de una $e(0)$ de 37.5 ; (gráfica $G$ ).

8) Una distorsión de la distribución por edad de las personas y las defunciones por la imposición de desviaciones proporcionales de la estabilidad que es encontrada en las mujeres de la India registradas en el censo de 1911. (gráfica $\mathrm{H}$ ).

Cualquiera de los tres métodos anteriormente descritos puede ser usado para calcular una tabla de vida. Una tabla basada en las tasas específicas de mortalidad requiere esencialmente un registro completo de las defunciones y de la población; cada uno de los otros dos cálculos empleados solamente parten de la información utilizada. El método de Preston, et al, hace uso de las distribuciones de las defun-

\footnotetext{
* La población estable fue tomada a partir de la tabla modelo de vida femenina, región Oeste $[\mathrm{e}(0)=50$ años] y una tasa de crecimiento del $2.5 \%$ (Coale y Demeny, 1966).
} 


\section{Figura 1}

\section{EFECTOS DE DISTORSIONES SOBRE TABLAS DE VIDA CALCULADAS A PARTIR DE LAS DISTRIBUCIONES POR EDAD DE LA POBLACIÓN Y DEFUNCIONES Y DE LAS TASAS ESPECÍFICAS DE MORTALIDAD}
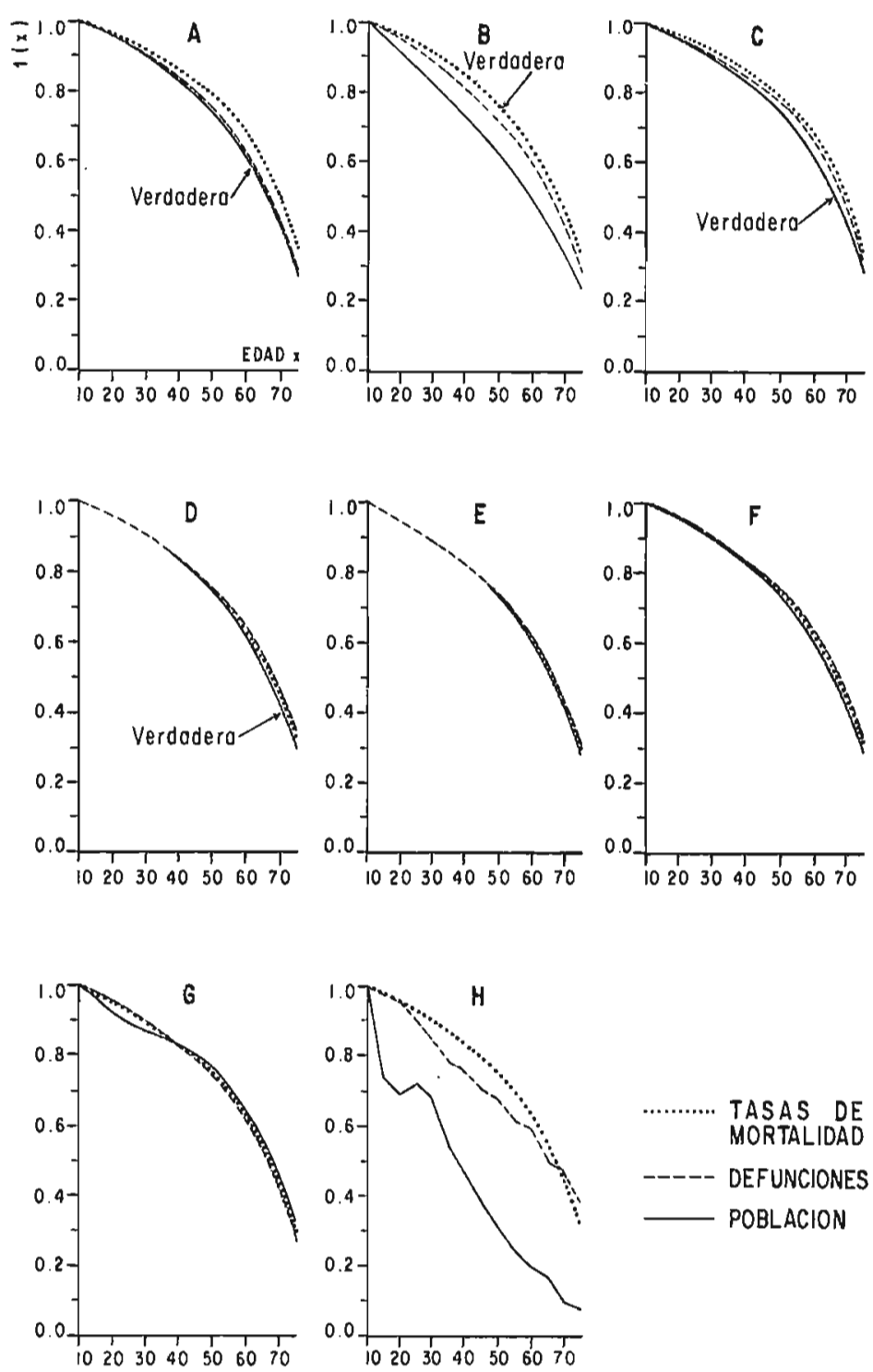
MORTALIDAD

----- DEFUNCIONES POBLACION

Fuente: Preston, et. 01. 0. 184. 
ciones y de la población por edad. Debido a la acumulación de las defunciones y personas de las edades avanzadas a edades tempranas, esto es relativamente robusto en la presencia del registro erróneo por edad.

El método depende de la igualdad, en cualquier cohorte no sujeta a ganancias o pérdidas por migración, del número de personas vivas a la edad $a$ y el número de defunciones que esas personas experimentarán hasta el último sobreviviente. En una población estable, el número de defunciones que serán experimentadas (bajo el riesgo constante de la mortalidad) puede ser estimado a partir de] número de defunciones ocurridas registradas a partir de la edad $a$. Específicamente, donde $N(a)$ es el número de personas a la edad $a$ en una población estable, y $D(x)$ es el número de defunciones a la edad $x$ :

$$
N(a)=\sum_{x=a}^{\omega} D(x) \exp [r(x-a)]
$$

$D(x)$ es el número de defunciones experimentadas por las personas con la edad $x$, sujetas a la tasa de mortalidad $\mu(x)$. Cuando las personas que ahora tienen la edad $a$ alcancen la edad $x$ ( $x-a$ años a partir de ahora) la población de edad $x$ habrá crecido en un factor exp $[r(x-a)]$, y con las tasas de mortalidad supuestas constantes, el número de defunciones de personas que tienen ahora la edad $a$ que experimentarán a la edad $x$ serán más grandes que el número progresivo por el mismo factor. Así, el lado derecho de la ecuación (11) es el número total de defunciones de personas de edad $a$ experimentadas si la mortalidad y la composición por edad permanecen fijas, y este número es precisamente igual, en una población estable, al número que ahora tiene la edad $a$.

$$
\text { Sea } \hat{N}(a)=\sum_{x=a}^{\omega} D(x) \exp [r(x-a)]
$$

una estimación del número de personas a la edad $a$, una estimación derivada a partir del número registrado de defunciones de todas las edades arriba de $a, y$ de la tasa de crecimiento. Si la población es genuinamente estable, la tasa de crecimiento estipulada es la correcta, y las defunciones y población registradas son las que precisamente se dan, entonces $\hat{N}(a)$ se igualará a $N(a)$. Si las defunciones están subregistradas en alguna proporción fija (digamos $20 \%$ ), $\hat{N}(a) / N(a)$ será menor que 1.0 (0.80 en este ejemplo). En la práctica se tendría que usar la aproximación discreta ${ }_{5} \hat{N}_{a} /{ }_{5} N_{a}$, la razón de la estimación para la población entre las edades $a$ y $a+5$.

E] método de Preston, et al., considera inapropiado estimar la integridad del registro de defunciones relativo a la enumeración de la población a partir de un único cociente de ${ }_{5} \hat{N}_{a} /{ }_{5} N_{a}$, donde el número en una edad particular está sujeto a sobreregistro o subregistro debido al reporte erróneo por edad o a omisión diferencial de personas en un conjunto particular de edades. Por lo que recomiendar el cálculo de $\hat{N}(a+) / N(a+)$ (donde $\hat{N}(a+)=\sum_{a}^{\omega}{ }_{5} \hat{N}_{x}$ ), para valores secuenciales de $a$ a partir de 5 o 10 a 60,70 u 80 . Con registro consistente de defunciones y población, mínimo error de registro por edad, y unà estimación válida de la tasa de crecimiento, $\hat{N}(a+) / N(a)$ sería aproximadamente constante cuando $a$ creciera, a un nivel promedio igual a la integridad del registro de defunciones relativo a la 
integridad de la enumeración de la población. Los autores muestran cómo la falta de estabilidad, error en el registro por edad, cobertura diferencial del registro de defunciones a edades diferentes, o error en la estimación de la tasa de crecimiento afectan la secuencia de los cocientes ${ }_{5} \hat{N}_{a} /{ }_{5} N_{a}$ y $\hat{N}(a+) / N(a+)$. Con lo que la predicción natural del efecto de defectos particulares en los datos sobre las secuencias es una herramienta de diagnóstico adicional para detectar ( $y$ algunas veces para corregir) los defectos.

En las gráficas de la figura 2 se observan los siguientes efectos sobre las series ${ }_{5} \hat{N}_{a} /{ }_{5} N_{a}$ y $\hat{N}(a+) / N(a+):$

a) una incorrecta estimación de $r$ (gráfica $A$ ); $b$ ) cobertura del registro de defunciones que varia con la edad (gráfica B); $c$ ) sobreestimación por edad de las defunciones (gráfica $C$ ); d) sobreestimación por edad de las personas (gráfica D); e) sobreestimación tanto de defunciones como de personas vivas (gráfica E); f) una población desestabilizada con una reciente historia de descenso de la mortalidad (gráfica $\mathrm{F}$ ) ; y $\mathrm{g}$ ) fuerte error en el registro por edad de personas y de defunciones del tipo que duplicaría la incorrecta distribución por edad de la población femenina de la India registrada en el censo de 1911 (gráfica G).

Otros de los puntos que muestran los autores, en cuanto a los efectos de las distorsiones se refiere, es con respecto a la tasa de "hatalidad" por encima de la edad $a[N(a) / N(a+)]$ y la tasa de mortalidad por encima de la edad $a[D(a+) / N(a+)]$. En la figura 3 se observan los siguientes efectos sobre las gráficas de $D(a+) / N(a+)$ contra $N(a) / N(a+)$, para una población estable hipotética: a) sin distorsiones (gráfica A); b) aumento de la integridad del registro con la edad (gráfica B); c) desplazamiento hacia adelante de la edad al morir (gráfica C); d) desplazamiento hacia adelante de la edad de la población (gráfica D); $e$ ) desplazamiento hacia adelante de las edades al morir y de la población (gráfica E); f) población sujeta a la disminución de la mortalidad en los últimos 25 años (gráfica F); y g) distorsiones del censo de la India, 1911 (gráfica G).

Finalmente los autores señalan que:

a) Desafortunadamente no es fácil distinguir entre algunas de las violaciones subyacentes de los supuestos. Por ejemplo, la selección de una tasa de crecimiento demasiado pequeña y un desplazamiento hacia arriba de las defunciones, producen un crecimiento de la secuencia de $\hat{N} / N$. En forma similar, desplazamientos hacia abajo de las defunciones (o integridad del registro que decrece con la edad) y una selección de $r$ demasiado grande producen una caida de la secuencia de $N / N$. Al encontrar una secuencia elevada, debe decidirse cuál de las dos distorsiones es más verosímil, para efectuar una corrección. Si se decide que $r$ es demasiado pequeña, simplemente se probará un valor mayor. Si las defunciones están desplazadas hacia arriba, puede hacerse una corrección similar.

b) Si la secuencia ${ }_{5} \hat{\mathrm{Na}}{ }_{5} \mathrm{Na}$ es muy irregular, y si los puntos $d(a+), b(a+)$ están alejados de lo lineal, los cálculos sintéticos sugieren que la mediana de los ocho primeros valores de $\hat{N}(a+) / N(a+)$ podrian proveer una estimación robusta de la cobertura. Ni las severas distorsiones de las distribuciones por edad ni la desestabilización causada por disminución reciente de la mortalidad introducen errores en la estimación de la cobertura que son superiores a algunos porcentajes.

A continuación se resume el método para calcular $\hat{N}$ : el problema característico del cálculo es el intervalo de edades avanzadas el así llamado "intervalo abier. 
Figura 2

\section{EFECTO DE DISTORSIONES SOBRE LOS VALORES DE N̂/N}
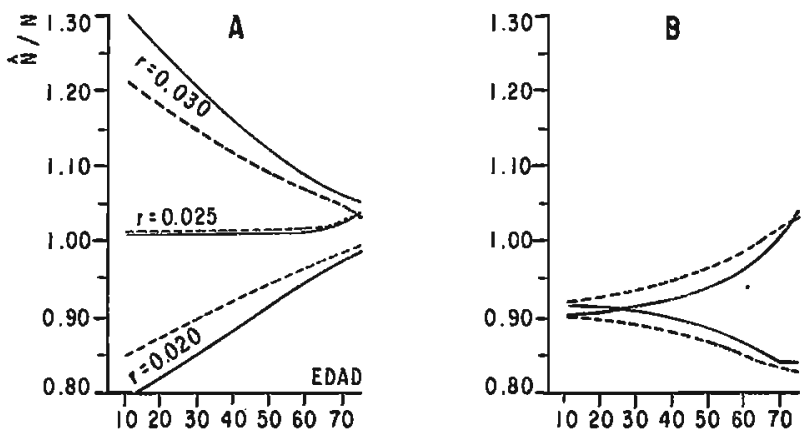

$\longrightarrow{ }_{5} \mathrm{~N}_{0} /{ }_{5} \mathrm{~N}_{0}$

$-\cdots-n \hat{N}(0+) / N(0+)$
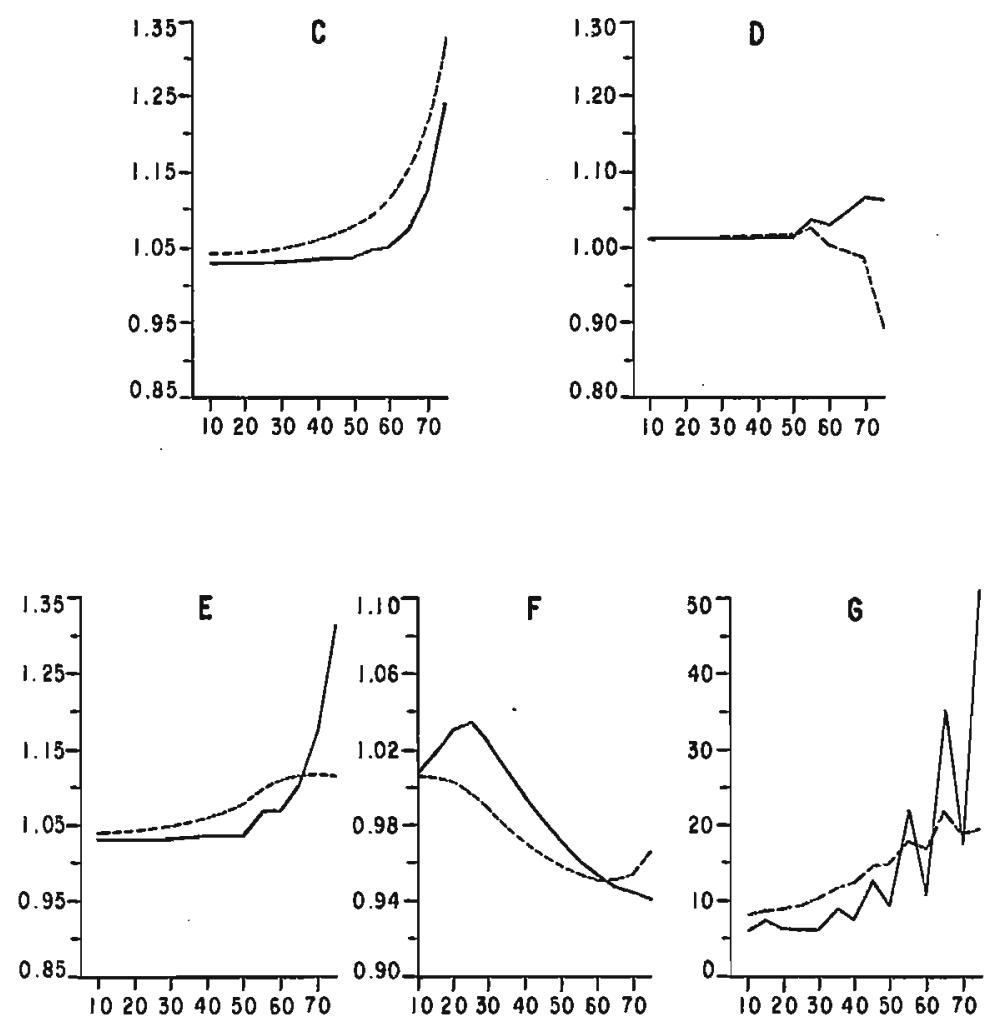

Fuente: Preston, et.al. p. 187. 
Figura 3

\section{EFECTO DE DISTORSIONES SOBRE LA GRÁFICA DE $\mathrm{D}(\mathrm{a}+) / \mathrm{N}(\mathrm{a}+)$ CONTRA $\mathrm{N}(\mathrm{a}) / \mathrm{N}(\mathrm{a}+)$}
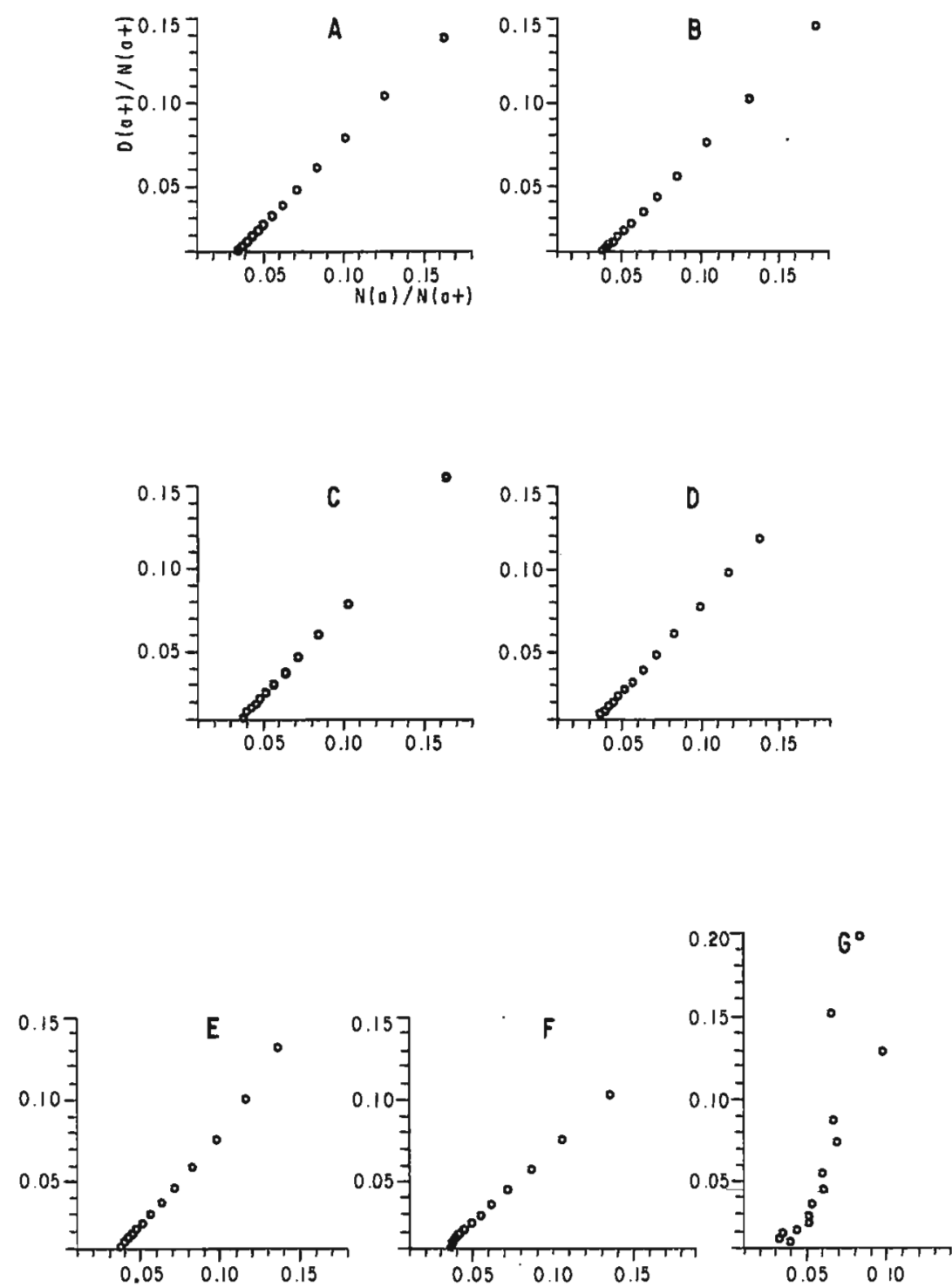

Fuente: Preston, et 01. 0. 191 
to". Si $A$ es el límite inferior del intervalo abierto, puede fácilmente verificarse que $\hat{N}(A) \doteq D(A+)[1+r e(A)]$, y $\hat{N}(A+) \doteq \hat{N}(A+) \cdot e(A)(1-r \bar{y})$, donde $e(A)$ es la esperanza de vida a la edad $A$, y $\bar{y}=$ [(edad media de la población estacionaria por encima de la edad $A$ ) $-A$ ]. Con información sobre mortalidad, desconocien. do la cobertura y estando sujeto a grandes márgenes de error de registro por edad en las edades avanzadas, no es posible calcular $e(A)$ y $\bar{y}$ a partir de dichos datos. Los autores sugieren tomar los valores aproximados de la siguiente tabla:

Tabla 1

\begin{tabular}{cccc}
\hline & $\begin{array}{c}\text { Mortalidad alta } \\
\left(\mathrm{e}_{10}=45\right) \\
\mathrm{e}(\mathrm{A})\end{array}$ & $\begin{array}{c}\text { Mortalidad media } \\
\left(\mathrm{e}_{10}=50\right) \\
\mathrm{e}(\mathrm{A})\end{array}$ & $\begin{array}{c}\text { Mortalidad baja } \\
\left(\mathrm{e}_{10}=55\right) \\
\mathrm{e}(\mathrm{A})\end{array}$ \\
\hline 65 & 9.9 & 11.2 & 12.3 \\
70 & 7.7 & 8.8 & 9.6 \\
75 & 5.9 & 6.7 & 7.3 \\
80 & 4.5 & 5.0 & 5.4 \\
85 & 3.3 & 3.6 & 3.8 \\
\hline
\end{tabular}

FUENTE: Preston et al. p. 183.

Esos valores son derivados de las tablas de vida modelo. Cálculos similares $y$ demuestran que puede ser aproximadamente cercana a $2 / 3 e(A)$. En poblaciones específicas, aproximadamente la esperanza de vida indicada a la edad 10, toma valores que podrían ser bastante diferentes. Con el fin de determinar los valores aproximados de $e(10)$ en una población, recomiendan calcular una tabla de vida basada en la distribución por edad de las defunciones y una estimación de $r$, puesto que dicha tabla de vida no estaría afectada por el subregistro uniforme de las defunciones. Podría sospecharse que algunas repeticiones de este proceso serían necesarias si cálculos subsecuentes revelaran que la selecciôn de $r$ es incorrecta o que las defunciones no son subregistradas uniformemente. Cálculos de prueba sugieren que dicho proceso iterativo no es necesario. Si $A$ es $75 \mathrm{o}$ más, errores en la estimación de $e(A)$ y $\bar{y}$ tienen poco efecto sobre $\hat{N}(a) \circ \hat{N}(a+)$ para edades menores de los 60 años. Una vez que los valores $\hat{N}(A)$ y $\hat{N}(A+)$ hayan sido calculados para el intervalo abierto por el empleo de los valores de $e(A)$ se estiman los valores $\hat{N}(a),{ }_{5} \hat{N}_{a-5}$ y $\hat{N}(a-5+)$ empleando las siguientes relaciones.

$\hat{N}(a-5)=\widehat{N}(a) \exp (5 r)+{ }_{5} D_{a-5} \exp (2.5 r)$

${ }_{3} N_{a-5}=2.5[\hat{N}(a)+\hat{N}(a-5)] a=A, A-5, A-10, \ldots 10$

$\hat{N}(a=5+)={ }_{5} \hat{N}_{a-5}+\hat{N}(a+)$ 
A continuación se ejemplifica el método descrito para estimar el grado de registro de la mortalidad adulta en México. Se tomó como información las estructuras por edad de la población y de las defunciones, de los hombres y mujeres en 1930. Para ello se recurrió al V Censo Nacional de Población y a las Estadísticas Vitales Mexicanas.

Las estructuras por edades antes mencionadas, se tomaron sin modificarlas, es decir, tal y como en las fuentes originales se daban. Para el cálculo de las tablas de vida se tomó como tasa de crecimiento, $r=0.01727$, la cual es una tasa de crecimiento intercensal; dicha tasa de crecimiento se utilizó para obtener la serie de los sobrevivientes, $l_{x}$, que se genera a partir de la estructura por edad de las defunciones. También se utilizó para obtener la población al 30 de junio de 1930, y con ella estimar la serie $l_{x}$ a partir de las tasas específicas de mortalidad. La esperanza de vida a los 80 años, necesaria para la aplicación del método, se tomó como 3.3 años; dicho valor es el sugerido por los autores suponiendo alta mortalidad y una esperanza de vida a los 10 años de 45 años.

En los cuadros 1 y 2 se sintetizan los cálculos de las tablas de vida, obtenidas a partir de las estructuras por edad de la población, defunciones y tasas de mortalidad, tanto para hombres como para mujeres en México, en 1930. En las gráficas 1 y 2 se muestran, para hombres y mujeres en 1930 en México, las distribuciones de la serie $l_{x}$ obtenida a partir de las distribuciones antes señaladas.

Los cuadros 3 y 4 resumen los cálculos llevados a cabo, para estimar el grado de registro de la mortalidad adulta en México, para hombres y mujeres en 1930; el comportamiento gráfico de la serie $D(a+) / N(a+)$ contra la serie $\hat{N}(a+) / N(a+)$, se muestran en las gráficas 3 y 4 para hombres y mujeres respectivamente; y en las gráficas 5 y 6 las distribuciones de las series ${ }_{5} \hat{N}_{a} / 5 N_{a}$ y $\hat{N}(a+) / N(a+)$.

Algunas de las consideraciones que se desprenden de la observación de las gráficas son: la tabla de vida que se genera a partir de la estructura por edad de la población, muestra para los hombres, una distribución semejante a la población sujeta a una disminución de la mortalidad en los últimos veinticinco años; y la tabla de vida obtenida a partir de la estructura por edad de la población para las mujeres muestra distorsiones, de los 10 a los 50 años semejantes a las de la India en su censo de 1911 , sólo que en este caso se tienen por encima de las series $l_{x}$ de defunciones y tasas, la serie $l_{x}$ de la población. Las gráficas 3 y $4[D(a+) / N(a+)$ contra $N(a) / N(a+)]$ muestran efectos de distorsiones semejantes a laś observadas en el censo de la India en 1911, tanto para los hombres como para las mujeres. Finalmente las gráficas 5 y 6 (distribuciones de ${ }_{5} \hat{N}_{a} /{ }_{5} N_{a}, \hat{N}(a+) / N(a+)$ ) tanto en el caso de los hombres como en el de las mujeres, el efecto de distorsión es seme. jante al que muestran los autores con una población que tiene un desplazamiento hacia arriba de la edad al morir y la edad de la población, aunque también es parecido al caso del censo de la India de 1911.

Cabe señalar que los autores también ejemplifican el método para casos como las mujeres de Brasil en 1961 y las mujeres de Corea en el período 1965-1975, en ambos casos, a diferencia del caso hombres y mujeres en 1930 en México, la tendencia de la serie $\hat{N}(a+) / N(a+)$ oscila alrededor de un solo valor, en el caso de las mujeres de El Salvador de 1961 alrededor de $0.8 \mathrm{y}$ el de las coreanas alrededor de 0.6. Así los autores adoptan como criterio, tomar la mediana de un conjunto de valores de la serie antes señalada, para el caso de los ejemplos presentados. 


\section{Cuadro 1}

MEXICO: CÁlCULO DE las TABLAS DE VIDA A PARTIR DE LAS ESTRUCTURAS POR EDAD DE LA POBLACióN, DEFUNCIONES Y TASAS DE MORTALIDAD, HOMBRES, 1930.

\begin{tabular}{|c|c|c|c|c|c|c|c|c|c|}
\hline Edad & ${ }_{5} \mathrm{~N}_{\mathrm{X}}^{\mathrm{a}}$ & ${ }_{5} \mathrm{~N}_{\mathrm{X}}^{\mathrm{m}^{\mathrm{b}}}$ & ${ }_{5} D_{X}{ }^{c}$ & ${ }_{5} L_{x}{ }^{d}$ & $1_{\mathrm{X}}^{\mathrm{P}^{\mathrm{e}}}$ & f & $1_{\mathrm{x}}^{\mathrm{D}^{\mathrm{g}}}$ & ${ }_{5} \mathrm{~m}_{\mathrm{x}}{ }^{\mathrm{h}}$ & $1_{X}^{M^{i}}$ \\
\hline 10 & 882039 & 883903 & 5169 & 4.48586 & 1.00000 & 199120.27680 & 1.00000 & 0.00585 & 1.00000 \\
\hline 15 & 792389 & 794064 & 5960 & 4.99792 & 0.94838 & 193723.22060 & 0.97290 & 0.00751 & 0.97117 \\
\hline 20 & 734715 & 736268 & 7737 & 4.44100 & 0.94389 & 186939.01220 & 0.93882 & 0.01051 & 0.93537 \\
\hline 25 & 687269 & 688721 & 8267 & 4.70048 & 0.91415 & 177337.78200 & 0.89061 & 0.01200 & 0.88748 \\
\hline 30 & 542978 & 544125 & 7706 & 3.90073 & 0.86012 & 166153.60570 & 0.83444 & 0.01416 & 0.83579 \\
\hline 35 & 500587 & 501645 & 8101 & 3.92052 & 0.78213 & 154788.18040 & 0.77736 & 0.01615 & 0.77863 \\
\hline 40 & 380027 & 380830 & 7186 & 3.24476 & 0.71653 & 141762.66350 & 0.71194 & 0.01887 & 0.71820 \\
\hline 45 & 313708 & 314371 & 6990 & 2.92007 & 0.61648 & 129166.25230 & 0.64868 & 0.02223 & 0.65349 \\
\hline 50 & 254878 & 255317 & 6583 & 2.58644 & 0.55065 & 115808.36230 & 0.58160 & 0.02577 & 0.58468 \\
\hline 55 & 163088 & 163433 & 5112 & 1.80423 & 0.43907 & 102093.66920 & 0.51272 & 0.03128 & 0.51390 \\
\hline 60 & 184768 & 185158 & 8186 & 2.22842 & 0.40327 & 90483.09035 & 0.45441 & 0.04421 & 0.43935 \\
\hline 65 & 90013 & 90203 & 4983 & 1.18352 & 0.34119 & 70213.89947 & 0.35262 & 0.05524 & 0.35190 \\
\hline 70 & 69849 & 69997 & 5627 & 1.00124 & 0.21848 & 56762.83910 & 0.28507 & 0.08039 & 0.26650 \\
\hline 75 & 36189 & 36265 & 3660 & 0.56552 & 0.15668 & 40203.47842 & 0.20191 & 0.10092 & 0.17730 \\
\hline 80 & 28265 & 28325 & 3793 & 0.48155 & 0.10471 & 28461.28342 & 0.14294 & 0.13391 & 0.10586 \\
\hline 85 & 17469 & 17506 & 3985 & 0.32445 & 0.08060 & 15194.92455 & 0.07631 & 0.22764 & 0.05276 \\
\hline
\end{tabular}

a Estructura por grupos quinquenales de edad observada, tomada del V Censo Nacional de Población.

b Estructura por grupos quinquenales de edad observada, proyectada al 30 de junio de 1930.

c Promedio de defunciones registradas en los años 1929-1931, por grupos quinquenales de edad.

d ${ }_{5} \mathrm{~L}_{\mathrm{x}}=\left({ }_{5} \mathrm{~N}_{\mathrm{x}} / \mathrm{N}(10)\right) \cdot(\exp (\mathrm{r}(\mathrm{x}-10+2.5)))$; donde $\mathrm{N}(10)=1 / 10\left({ }_{5} \mathrm{~N}_{5}+{ }_{5} \mathrm{~N}_{10}\right)$

e ${ }_{1}^{P}=1 / 10\left({ }_{5} L_{X-5}+{ }_{5} L_{x}\right)$

f Valores de $\sum_{x}^{80}\left|{ }_{5} D_{x} \cdot \exp (r(x-10+2.5))+D(85+) \exp (r(75+e(85)))\right|$

$\mathrm{g} \quad \mathrm{I}_{\mathrm{x}}^{\mathrm{D}}=\mathrm{Cx}(\mathrm{f})$ donde: $\mathrm{C}=1 /\left.\sum_{10}^{80}\right|_{5} \mathrm{D}_{\mathrm{x}} \cdot \exp (\mathrm{r}(\mathrm{x}-10+2.5))+\mathrm{D}(85+) \exp (\mathrm{r}(75+\mathrm{e}(85))) \mid$

h ${ }_{5} \mathrm{~m}_{\mathrm{X}}={ }_{5} \mathrm{D}_{\mathrm{X}} / \mathrm{N}_{5} \mathrm{~m}$

i $\quad 1_{x-5}^{M}=1_{x}^{M}\left(1-{ }_{5} q_{x}\right) ;$ donde ${ }_{5} q_{x}=\left(5 \cdot{ }_{5} m_{X}\right) /\left(1+2.55 m_{x}\right)$ 
Cuadro 2

México: Cálculo de las tablas de vida a partir de las estructuras por edad de la población, DEFUNCIONES Y TASAS DE MOR TALIDAD, MUJERES, 1930.

\begin{tabular}{|c|c|c|c|c|c|c|c|c|c|}
\hline Edad & a & $\mathrm{b}$ & c & d & e & f & $\mathrm{g}$ & h & $i$ \\
\hline$x$ & ${ }_{5} \mathrm{~N}_{x}$ & ${ }_{5} \mathrm{~N}_{x}^{m}$ & ${ }_{5} \mathrm{D}_{\mathrm{x}}$ & $5 L_{x}$ & $1_{x}^{P}$ & & $\mathbf{l}_{x}^{\mathrm{D}}$ & ${ }_{5} \mathrm{~m}_{\mathrm{x}}$ & $1 \frac{\mathrm{M}}{\mathrm{M}}$ \\
\hline 10 & 804025 & 805724 & 4459 & 4.35300 & 1.00000 & 200180.675 & 1.00000 & 0.00553 & 1.00000 \\
\hline 15 & 892277 & 894163 & 6514 & 5.26648 & 0.96195 & 195524.944 & 0.97674 & 0.00729 & 0.97273 \\
\hline 20 & 842218 & 843998 & 8043 & 5.41935 & 1.06858 & 188110.123 & 0.93970 & 0.00953 & 0.93795 \\
\hline 25 & 773330 & 774964 & 8357 & 5.42487 & 1.08442 & 178129.162 & 0.88984 & 0.01078 & 0.89430 \\
\hline 30 & $581 \quad 105$ & 582333 & 7369 & 4.44405 & 0.98689 & 166823.227 & 0.83336 & 0.01265 & 0.84736 \\
\hline 35 & 528873 & 529991 & 7368 & 4.40936 & 0.88534 & 155954.836 & 0.77907 & 0.01390 & 0.79541 \\
\hline 40 & 427258 & 428161 & 6417 & 3.88345 & 0.82928 & 144107.902 & 0.71989 & 0.01499 & 0.74198 \\
\hline 45 & 321042 & 321720 & 5743 & 3.18120 & 0.70647 & 132859.479 & 0.66370 & 0.01785 & 0.68838 \\
\hline 50 & 289495 & $290 \quad 107$ & 58 & 32 & 085 & 884.606 & 0.60887 & 29 & 0.62956 \\
\hline 55 & 162441 & 162784 & 4567 & 1.91304 & 0.50404 & 109624.091 & 0.54763 & 0.02806 & 0.56880 \\
\hline 60 & 198588 & 199008 & 8050 & 2.54967 & 0.44627 & 99251.338 & 0.49581 & 0.04045 & 0.49426 \\
\hline 65 & 85809 & 85990 & 4934 & 1.20107 & 0.37507 & 79318.894 & 0.39624 & 0.05738 & 0.40347 \\
\hline 70 & 74771 & 74929 & 5876 & 1,14094 & 0.23420 & 66000.104 & 0.32970 & 0.07842 & 0.30224 \\
\hline 75 & 33856 & 33928 & 3676 & 0.56321 & 0.17042 & 48707.976 & 0.24332 & 0.10835 & 0.20315 \\
\hline 80 & 32313 & 32381 & 4616 & 0.58602 & 0.11492 & 36914.449 & 0.18441 & 0.14255 & 0.11656 \\
\hline 85 & 20211 & 20254 & 5447 & 0.40517 & 0.09912 & 20769.574 & 0.10375 & 0.26893 & 0.05531 \\
\hline
\end{tabular}

Fistructura por grupos quinquenales de edad observada, lomada del $V$ Censo Nacional de Población.

Estructura pur grupos quinquenales de edad ubservada, proyectada al 30 de junio de 1930.

c Promedio de defunciones registradas cn los años 1929-1931, por grupos quinquenales de edad.

d ${ }_{5} L_{x}=\left({ }_{s} N_{x} / N(10)\right) \cdot(\exp (r(x-10+2.5))):$ donde $N(10)=1 / 10\left({ }_{s} N_{s}+{ }_{s} N_{10}\right)$

e $I_{x}^{P}=1 / 10\left(s L_{x-s}+s L_{x}\right)$

f Valores de $\sum_{x}^{80}\left|{ }_{5} D_{\mathbf{x}} \cdot \exp (r(x-10+2.5))+D(85+) \exp (r(75+e(85)))\right|$

g $I_{x}^{D}=C x(f)$ donde: $C=1 /\left.\sum_{10}^{80}\right|_{s} D_{x} \cdot \exp (r(x-10+2.5))+D(85+) \exp (r(75+e(85))) \mid$

h ${ }_{5} m_{x}={ }_{5} D_{x} /{ }_{5} N_{x}^{m}$

i $\quad l_{x-5}^{M}=1_{x}^{M}\left(1-{ }_{5} q_{x}\right) ;$ donde ${ }_{5} q_{x}=\left(5 \cdot{ }_{5} m_{x}\right) /\left(1+2.5{ }_{5} m_{x}\right)$ 


\section{Cuadro 3}

MÉXICO: DEFUNCIONES Y POBLACIÓN POR EDAD Y VALORES DE $\hat{\mathrm{N}}(\mathrm{a}),{ }_{5} \hat{\mathrm{N}}_{a}, \hat{\mathrm{N}}(a+),{ }_{5} \hat{\mathrm{N}}_{a} /{ }_{5} \mathrm{Na}$ y $\hat{\mathrm{N}}(\mathrm{a} a) / \mathrm{N}(\mathrm{a}+)$, HOMBRES, 1930

\begin{tabular}{|c|c|c|c|c|c|c|c|c|}
\hline Edad & ${ }_{5} \mathrm{D}_{\mathrm{a}}$ & ${ }_{5} \mathrm{~N}_{\mathrm{a}}$ & $N(a+)$ & $\hat{\mathrm{N}}(\mathrm{a})$ & ${ }_{5} \hat{\mathrm{N}}_{\mathrm{a}}$ & $\hat{N}(\mathrm{a}+)$ & ${ }_{5} \mathrm{~N}_{\mathrm{a}} / 5 \mathrm{Na}$ & $\hat{N}(a+) / N(a+)$ \\
\hline 0 & 113633 & 1268968 & 8115935 & 370827 & 1505363 & 8396739 & 1.186 & 1.135 \\
\hline 5 & 13425 & 1168736 & 6846967 & 231318 & 1076600 & 6891376 & 1.921 & 1.006 \\
\hline 10 & 5169 & 882039 & 5678231 & 199322 & 943005 & 5814776 & 1.069 & 1.024 \\
\hline 15 & 5960 & 792389 & 4796192 & 177880 & 838338 & 4871771 & 1.058 & 1.016 \\
\hline 20 & 7737 & 734715 & 4003803 & 157455 & 736183 & 4033433 & 1.002 & 1.007 \\
\hline 25 & 8267 & 687269 & 3269088 & 137018 & 636955 & 3297250 & 0.927 & 1.009 \\
\hline 30 & 7706 & 542978 & 2581819 & 117764 & 546010 & 2660295 & 1.006 & 1.030 \\
\hline 35 & 8101 & 500587 & 2038841 & 100640 & 462988 & 2114285 & 0.925 & 1.037 \\
\hline 40 & 7186 & 380027 & 1538254 & 84.555 & 388080 & 1651297 & 1.021 & 1.073 \\
\hline 45 & 6990 & 313708 & 1158227 & 70677 & 322030 & 1263217 & 1.027 & 1.091 \\
\hline 50 & 6583 & 254878 & 844519 & 58135 & 262890 & 941187 & 1.031 & 1.114 \\
\hline 55 & 5112 & 163088 & 589641 & 47021 & 213138 & 678297 & 1.307 & 1.150 \\
\hline 60 & 8186 & 184768 & 426553 & 38238 & 163663 & 465159 & 0.886 & 1.091 \\
\hline 65 & 4983 & 90013 & 241785 & 27231 & 118593 & 301496 & 1.318 & 1.247 \\
\hline 70 & 5627 & 69849 & 151772 & 20206 & 83378 & 182903 & 1.194 & 1.205 \\
\hline 75 & 3660 & 36189 & 81923 & 13145 & 54243 & 99525 & 1.499 & 1.215 \\
\hline 80 & 3793 & 28265 & 45734 & 8552 & 31910 & 45282 & 1.129 & 0.990 \\
\hline 85 & 3985 & 17369 & 17469 & 4212 & 13372 & 13372 & 0.765 & 0.765 \\
\hline
\end{tabular}




\section{Cuadro 4}

MÉXICO: DEFUNCIONES Y POBLACIÓN POR EDAD Y VALORES DE $\hat{\mathbf{N}}(a),{ }_{5} \hat{\mathrm{N}}_{\mathrm{a}}, \hat{\mathrm{N}}(\mathrm{a}+),{ }_{5} \hat{\mathrm{N}}_{\mathrm{a}} /{ }_{5} \mathrm{~N}_{\mathrm{a}}$ y $\hat{\mathrm{N}}(\mathrm{a}+) / \mathrm{N}(\mathrm{a}+)$, MUJERES, 1930

\begin{tabular}{|c|c|c|c|c|c|c|c|c|}
\hline Edad & ${ }_{5} D_{a}$ & ${ }_{5} \mathrm{~N}_{\mathrm{a}}$ & $N(a+)$ & $\hat{N}(a)$ & ${ }_{5} \hat{\mathrm{N}}_{\mathrm{a}}$ & $\hat{N}(a+)$ & ${ }_{5} \hat{N}_{a} / 5 N_{a}$ & $\hat{N}(a+) / N(a+)$ \\
\hline 0 & 104663 & 1241553 & 8433694 & 362467 & 1486128 & 8547217 & 1.197 & 1.013 \\
\hline 5 & 12883 & 1124529 & 7192141 & 231984 & 1081090 & 7061089 & 0.961 & 0.982 \\
\hline 10 & 4459 & 804025 & 6067612 & 200452 & 950120 & 5979999 & 1.182 & 0.986 \\
\hline 15 & 6514 & 892277 & 5263587 & 179596 & 845238 & 5.029879 & 0.947 & 0.956 \\
\hline 20 & 8043 & 842218 & 4371310 & 158499 & 740453 & 4184641 & 9.879 & 0.957 \\
\hline 25 & 8357 & 773330 & 3529092 & 137682 & 639923 & 3444188 & 0.827 & 0.976 \\
\hline 30 & 7369 & 581105 & 2755762 & 118287 & 549325 & 2804265 & 0.945 & 1.018 \\
\hline 35 & 7368 & 528873 & 2174657 & 101443 & 468590 & 2254940 & 0.886 & 1.037 \\
\hline 40 & 6417 & 427258 & 1645784 & 85993 & 396815 & 1786350 & 0.929 & 1.085 \\
\hline 45 & 5743 & 321042 & 1218526 & 72733 & 334870 & 1389535 & 1.043 & 1.140 \\
\hline 50 & 5885 & 289495 & 897484 & 61215 & 279323 & 1054665 & 0.965 & 1.175 \\
\hline 55 & 4567 & 162441 & 607989 & 50514 & $231 \quad 185$ & 775342 & 1.423 & 1.275 \\
\hline 60 & 8050 & 198588 & 445548 & 41960 & 181848 & 544157 & 0.916 & 1.221 \\
\hline 65 & 4934 & 85809 & 246960 & 30779 & 135715 & 362309 & 1.582 & 1.467 \\
\hline 70 & 5876 & 74771 & 161151 & 23507 & 98605 & 226594 & 1.319 & 1.406 \\
\hline 75 & 3676 & 33856 & 86380 & 15935 & 67578 & 127989 & 1.996 & 1.482 \\
\hline 80 & 4616 & 32313 & 52524 & 11096 & 42133 & 60411 & 1.304 & 1.150 \\
\hline 85 & 5447 & 20211 & 20211 & 5757 & 18278 & 18278 & 0.904 & 0.904 \\
\hline
\end{tabular}




\section{Gráfica 1}

MÉXICO: TABLAS DE VIDA, HOMBRES, 1930

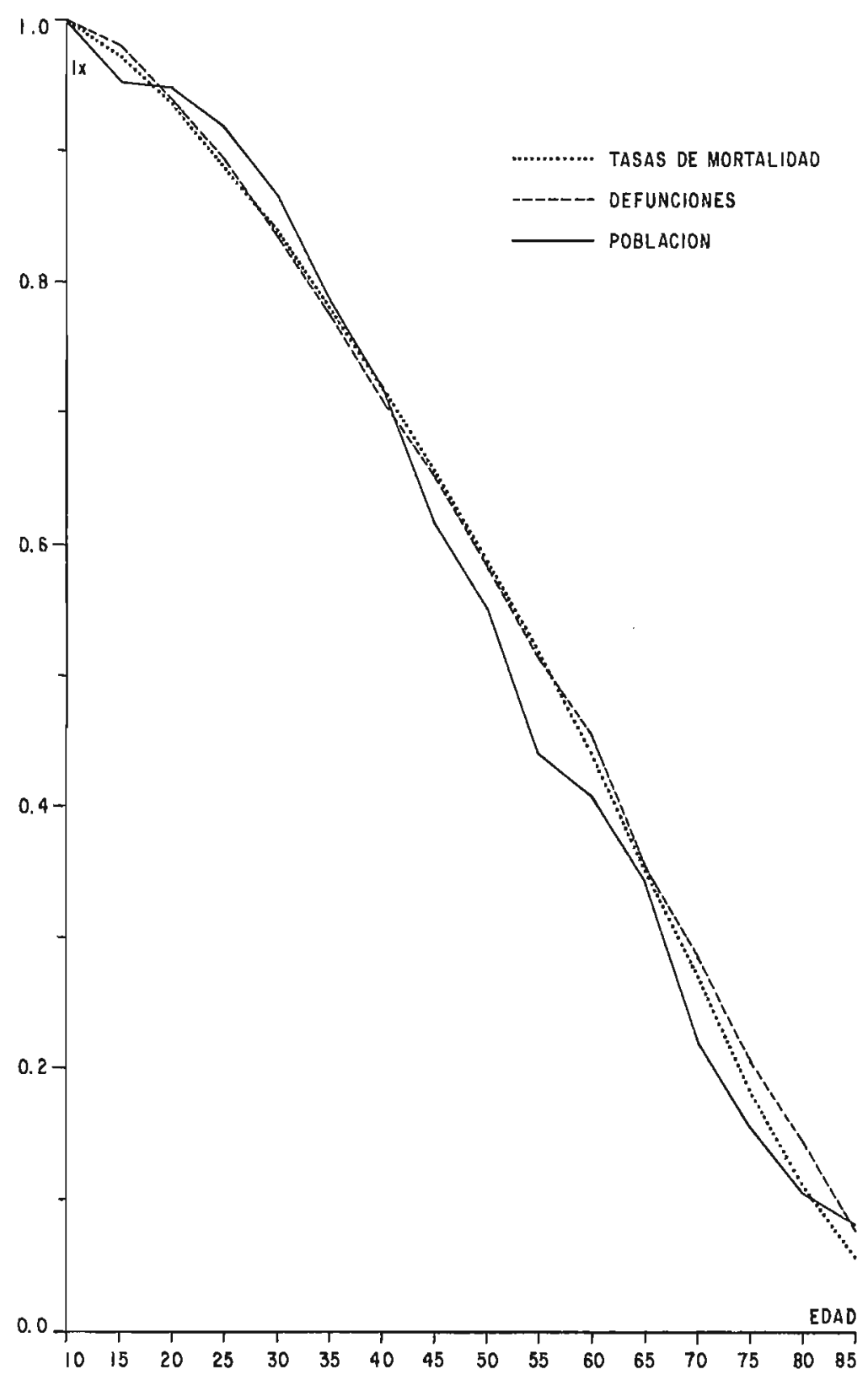




\section{Gráfica 2}

MÉXICO: TABLAS DE VIDA, MUJERES, 1930

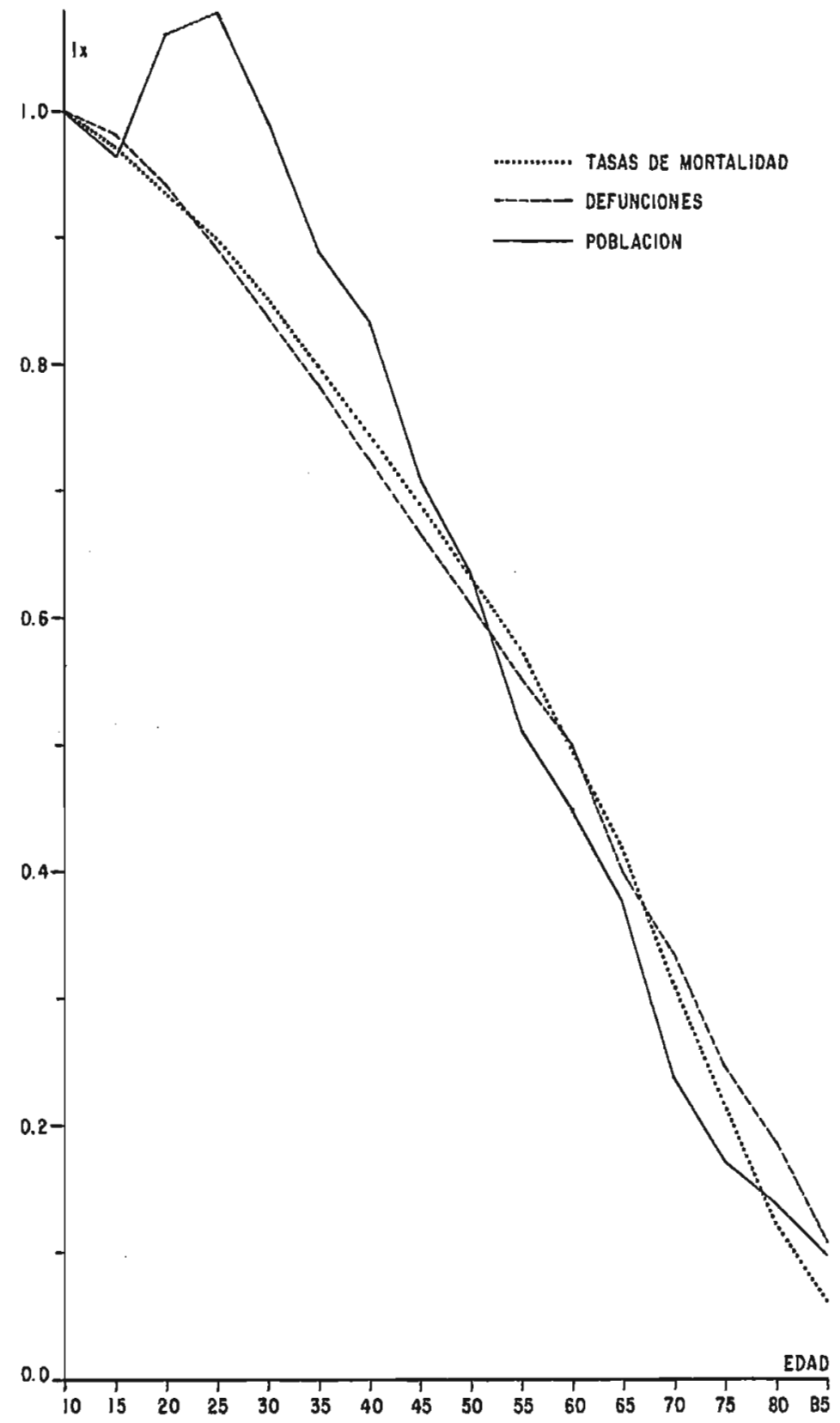




\section{Gráfica 3}

MÉXICO: GRÁfICA DE D(a+)/N(a+) CONTRA N(a+)/N(a+), hombres, 1930

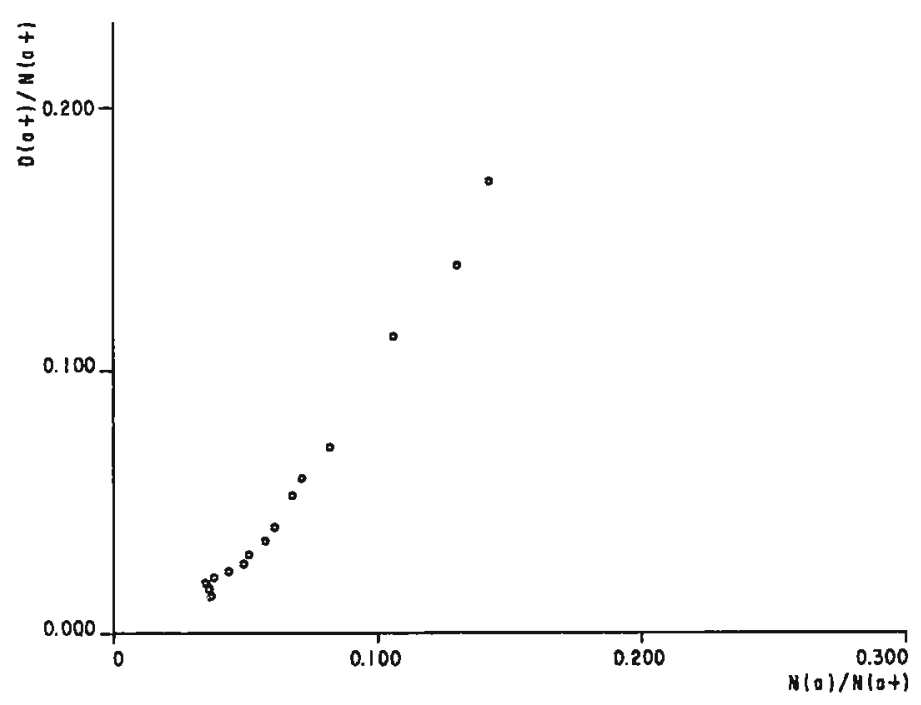

\section{Gráfica 4}

MÉXICO: GRÁFICA DE D(a+)/N(a+) CONTRA N(a)/N(a+) mujeres, 1930

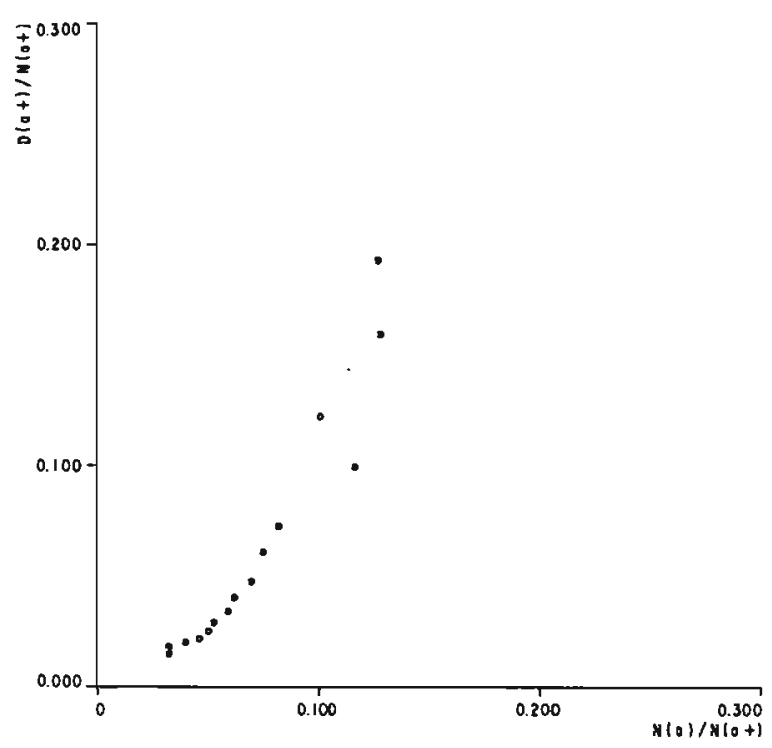




\section{Gráfica 5}

MÉXICO: GRÁFICA DE N̂/N, HOMBRES, 1930

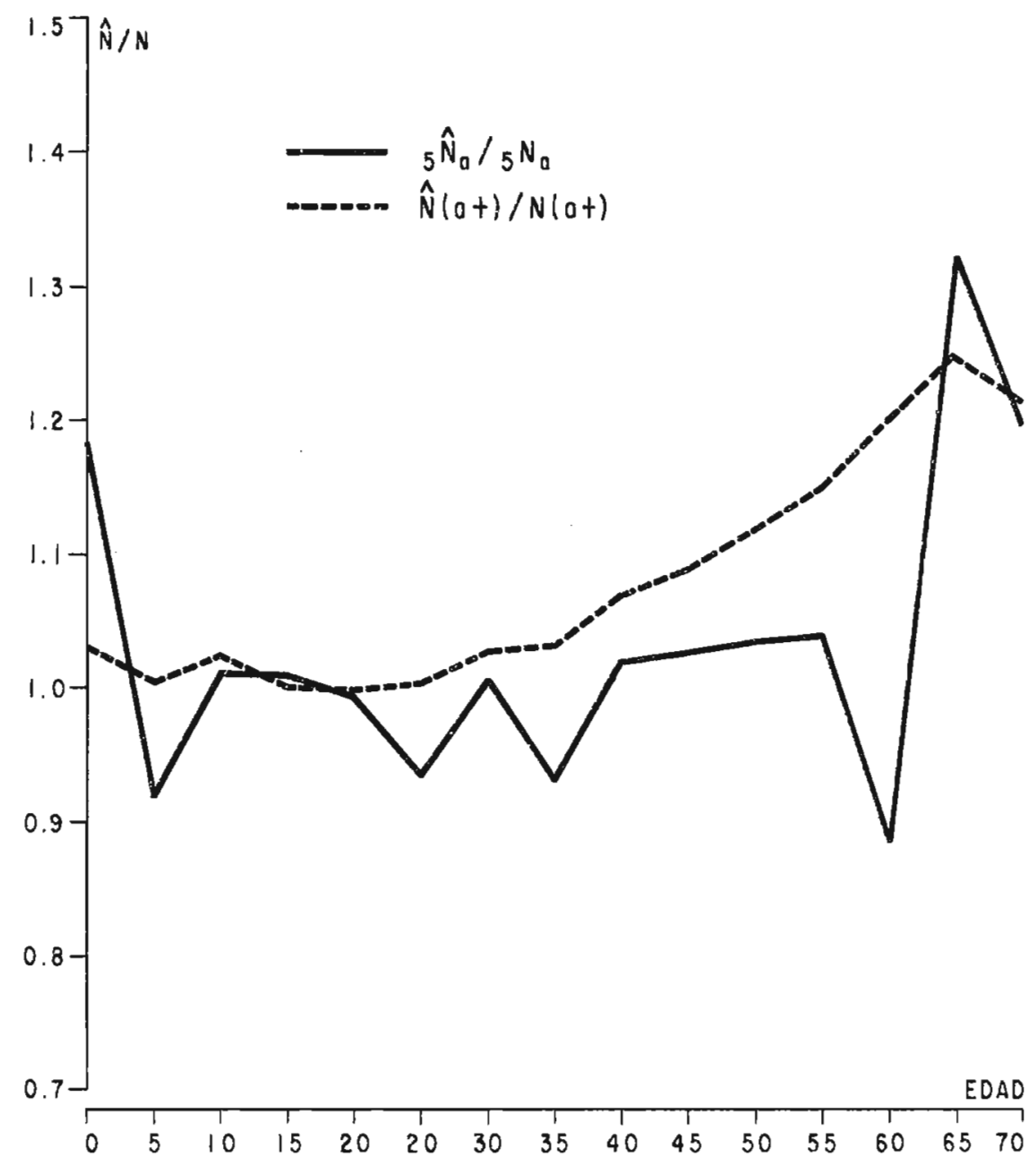




\section{Gráfica 6}

MÉXICO: GRÁFICA DE $\hat{\mathrm{N}} / \mathrm{N}$, MUJERES, 1930

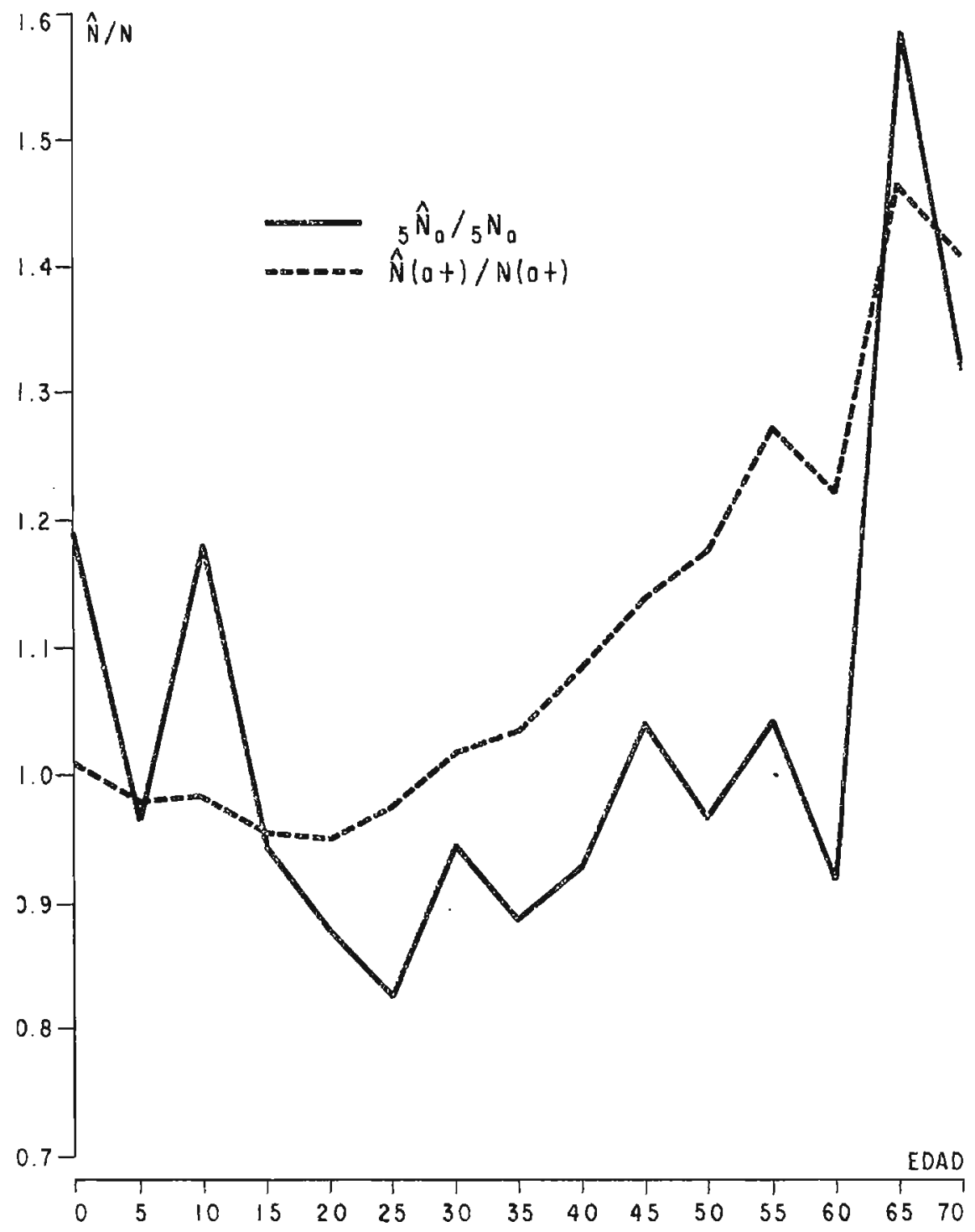


En el caso de México, tanto para hombres como para mujeres en 1930, la tendencia de la serie, $\widehat{N}(a+) / N(a+)$, no es homogénea además de mostrar, en general, un grado de sobreregistro de la mortalidad adulta, lo que contradice lo común. mente planteado, y esto debido a los efectos de las distorsiones mostradas, tanto en la construcción de las tablas de vida, como en las series que estiman el grado de subregistro de la mortalidad adulta.

A manera de conclusión se puede señalar que, en esta primera etapa de la inverstigación se observan serias distorsiones, sobre todo en las estructuras po: edades de las poblaciones censadas, desde el período 1930 hasta 1980. Para darse cuenta de la magnitud de las distorsiones, basta ver la gráfica 9 que se presenta en el apéndice 1 del presente trabajo, que muestra las distribuciones de las series $l_{x}$, obtenidas a partir de las distribuciones por edad de las tasas de mortalidad, defunciones y población

En e] apéndice número 2 se muestran las gráficas y cuadros de los valores ${ }_{5} \hat{N}_{a} /{ }_{5} N_{a}, \hat{N}(a+) \mid N(a+)$, por sexo, de 1940 a 1970 . Dichos valores muestran distorsiones semejantes a las observadas para el caso de 1930. Con el fin de diagnosticar el tipo y la magnitud de dichas distorsiones se observaron las gráficas y los valores de los parámetros $N(x) / N(x+)$ y $D(x+) / N(x+)$, por sexo, y para el mismo perfodo, 1940-1970; dichas gráficas y valores tambiến se presentan en el apéndice 2.

Después de analizar los resultados anteriormente señalados, se observó que una posible distorsión se debía al desplazamiento de las edades, tanto de la población como de las defunciones; por ello, como un primer intento de corregir dichas distorsiones, se calcularon y graficaron los valores $\hat{N}(x+5)+) / N(N x+)$ por' sexo, para el periodo 1930-1970 (véase apéndice 3), observándose en general valores más consistentes con la realidad mexicana, ya que se tienen, por un lado valores que muestran grados de subregistro (no como en el caso en que se tenían captados valores que mostraban sobreregistro) y por otro lado, el grado de subregistro es mayor para edades avanzadas. Cabe resaltar que, en general, el grado de subregistro es mayor para el caso de los hombres (véase gráfica 14 del apéndice 3).

Uno de los más recientes aportes sobre el tema de la medición de la integridad del registro de defunciones adultas, fue el hecho por Neil G. Bennett y Shiro Horiuchi, ${ }^{*}$ quienes parten del empleo de la relación obtenida para poblaciones estables, y usada por Preston y sus colegas.

$$
N(a)=\int_{a}^{\infty} D^{*}(x) \exp |r(x-a)| d_{x}
$$

Siendo la fórmula con la cual se estima la distribución por edad de la población:

$\hat{N}(a-5)=\hat{N}(a) \exp |5 r|+{ }_{5} D_{a-5} \exp |2.5 r|$

El método de Preston, et al. (1980) es claramente apropiado para el análisis de

* Bennett, G. Neil y Horiuchi, Shiro; 1981. "Estimating the completeness of Death Registration in a Closed Population". Population Index (Princeton, N.J.), 47 (2): 207-222. 
poblaciones estables, no obstante, para Bennett y Horiuchi, no es robusto en el contexto de la desestabilización.

Bennett y Horiuchi señalan que cuando una población se desvía de la estabilidad, la tasa de crecimiento natural ( $r$ ) varía con la edad, en cuyo caso, la tasa de crecimiento natural del total de la población es a menudo una pobre aproximación de $r(a)$, la tasa de crecimiento de la población a la edad $(a)$.

En base a lo anteriormente señalado, los autores proponen la siguiente extensión de la ecuación (2):

$\hat{N}(a-5)=\hat{N}(a) \exp \left|5_{5} r_{a-5}\right|+{ }_{5} D_{a^{-5}} \exp \left|2_{5.5} r_{a^{-5}}\right|$,

donde ${ }_{5} r_{a}-5$ es la tasa de crecimiento experimentada por la población que se en. cuentra entre las edades $a-5$ y $a$.

Ya calculados los valores de $\hat{N}(a)$ son calculados los valores ${ }_{5} \hat{N}_{a}$ de igual forma que en el método de Preston, et al.(1980):

$$
{ }_{5} \hat{N}_{a}=2.5|\hat{N}(a)+\hat{N}(a+5)|
$$

Al tratar la estimación del intervalo abierto los autores señalan que el método de Preston, et al., no es necesariamente adecuado en los casos en que se tiene un número limitadamente bajo de defunciones y en edades relativamente avanzadas, siendo en este caso $N(a)$ subestimada. Los autores encuentran la siguiente expresión para estimar la población en e] intervalo abierto:

$$
N(a) \doteq D(a+)\left[\exp |r(a+) e(a)|-\left(\cdot|r(a+) e(a)|^{2}\right)\right]
$$

Donde $r(a+)$ es la tasa de crecimiento para el intervalo abierto, y $e(a)$ la esperanza de vida al comienzo del intervalo abierto.;

De igual forma que en el caso de Preston, et al., en este caso se estiman, los valores $N(a)$ usando iterativamente la expresión (3).

Finalmente, los autores aplican su método al caso de Suecia y Corea, este último país también fue tomado por Preston, et al (1980), observando una mayor precisión de la estimación de] nivel de integridad del registro de defunciones adultas en poblaciones desestabilizadas.

En nuestro caso, inicialmente ejemplificamos e] método de Bennett y Horiuchi para el caso de México, hombres: período 1930-1935. En el cuadro 5 se resume el procedimiento por medio de] cual se estiman los valores ${ }_{10} N_{a-5} /{ }_{10} N_{a-5}, \mathrm{y}$ en la gráfica 7 se dan las secuencias de las estimaciones de la integridad del registro de defunciones, con y sin el supuesto de estabilidad, es decir, empleando el método de Preston, et al. y el de Bennett y Horiuchi; finalmente, en la gráfica 8 se muestran las distribuciones de los valores ${ }_{5} N_{a}$, observados y estimados. Después de ser analizadas las gráficas y los valores antes mencionados se observó que la "ganancia" que se lograba, al tomar tasas de crecimiento específicas por edad, no era significativa, observándose el mismo tipo de distorsiones que en el empleo de la tasa de crecimiento constante. Asi, se pasó a calcular y graficar los valores ${ }_{10} \hat{N}_{a / 10} N_{a^{-}}$, para los hombres en 1930-1935, (véase gráfica 15 en el apéndice 3 ). 


\section{Cuadro 5}

MÉXICO, DEFUNCIONES, POBLACIÓN, TASA DE CRECIMIENTO POR EDAD, Y VALORFS DE

$\hat{\mathrm{N}}(\mathrm{a}),{ }_{5} \hat{\mathrm{N}}_{\mathrm{a}} \mathrm{Y}_{10} \mathrm{~N}_{\mathrm{a}-5}$, HOMBRI:S, 1930-1935

\begin{tabular}{|c|c|c|c|c|c|c|}
\hline Edad & ${ }_{5} r_{a}$ & ${ }_{5} \mathrm{D}_{\mathrm{a}}$ & ${ }_{5} \mathrm{Na}_{\mathrm{a}}$ & $\hat{N}(a)$ & ${ }_{5} \hat{\mathrm{N}}_{\mathrm{a}}$ & ${ }_{10} \hat{N}_{a-5} / 10 \mathrm{~N}_{\mathrm{a}-5}$ \\
\hline 0 & .0139 & 113633 & 1268968 & 391270.40 & 1616376 & $-\ldots$ \\
\hline 5 & .0219 & 13425 & 1168736 & 255280.08 & 1178306 & 1.146 \\
\hline 10 & .0363 & 5169 & 882039 & 216042.37 & 978768 & 1.052 \\
\hline 15 & .0211 & 5960 & 792389 & 175363.81 & 819362 & 1.074 \\
\hline 20 & .0010 & 7737 & 734715 & 152279.90 & 720897 & 1.009 \\
\hline 25 & .0096 & 8267 & 687269 & 136079.09 & 644329 & 0.960 \\
\hline 30 & .0163 & 7706 & 542978 & 121652.70 & 565936 & 0.984 \\
\hline 35 & .0306 & 8101 & 500587 & 104721.70 & 467754 & 0.991 \\
\hline 40 & .0177 & 7186 & 380027 & 82379.93 & 377200 & 0.960 \\
\hline 45 & .0152 & 6990 & 313708 & 68500.06 & 313135 & 0.995 \\
\hline 50 & .0116 & 6583 & 254878 & 56753.89 & 259762 & 1.008 \\
\hline 55 & .0242 & 5112 & 163088 & 47150.93 & 210264 & 1.125 \\
\hline 60 & .0109 & 8186 & 184768 & 36954.81 & 159952 & 1.064 \\
\hline 65 & .0221 & 4983 & 90013 & 27026.14 & 116265 & 1.005 \\
\hline 70 & .0129 & 5627 & 69849 & 19479.96 & 80723 & 1.232 \\
\hline 75 & .0202 & 3660 & 36189 & 12809.06 & 52273 & 1.254 \\
\hline 80 & .0032 & 37.93 & 28265 & 8100.12 & 30774 & 1.288 \\
\hline 85 & .0169 & 3985 & 17469 & 4209.43 & -- & -- \\
\hline
\end{tabular}




\section{Gráfica 7}

MÉxico: SECuENCIA DE LAS ESTIMACiONES DE LA INTEGRIDAD DEL REGISTRO DE DEFUNCIONES, CON Y SIN EL SUPUESTO DE ESTABILIDAD, HOMBRES, 1930-1935

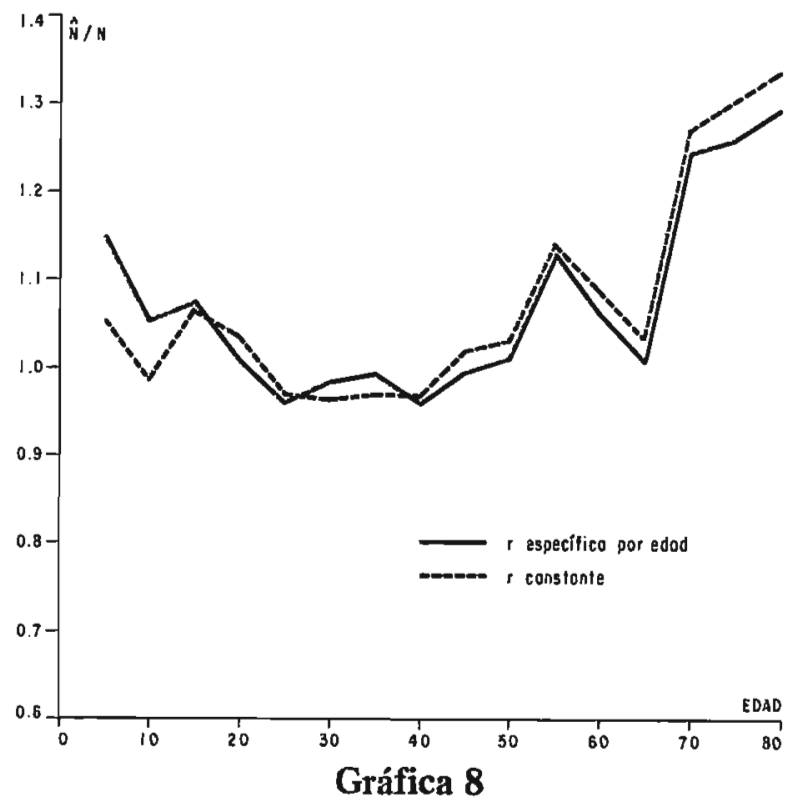

MÉXICO: DiSTRIBUCIONES DE LOS VALORES ${ }_{5} N_{2}$, OBSERVADOS Y ESTIMADOS, HOMBRES, 1930-1935

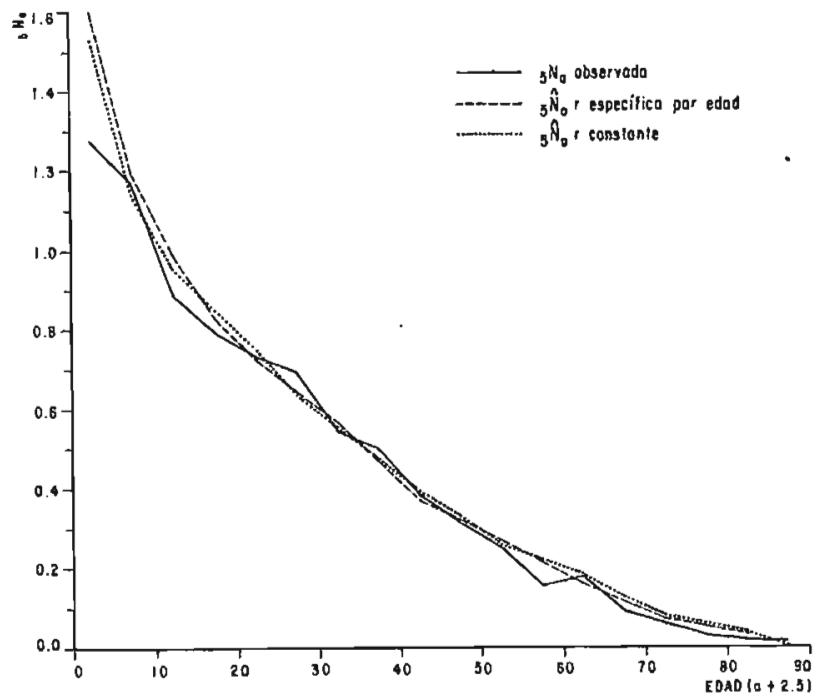


Finalmente, se obtuvieron las poblaciones estables asociadas a las poblaciones observadas* (por sexo, 1930-1970), calculándose los valores $\hat{N}[(x+5)+] /$ est.

$N(x+)$, donde $\hat{N}[(x+5)+]$ representa la población estimada por encima de la edad $x+5$ y $N(x+)$ es la población estable asociada con edad arriba de $x$. Los resultados se presentan en el cuadro 10 y en la gráfica 16 del apéndice 4.

En este caso se observa: $a$ ) que el grado de subregistro disminuye a través del tiempo, teniéndose una cobertura casi completa para $1970 ; b$ ) el subregistro es mayor para los hombres, previamente obtenidos, es difícil señalar un solo valor para resumir el grado de subregistro, para cada momento y sexo, por lo que se re. comienda tomar los resultados obenidos, por edad específica y tomando en cuenta el diagnóstico hecho previamente.

\section{CONCLUSIONES}

La cuantificación del grado de subregistro de la mortalidad adulta en México ha sido el objetivo central del presente trabajo. Se ha aplicado el método de Preston, et al. con el cual, en una primera parte se ha diagnosticado la calidad y tipo de información que se requieren para estimar el grado de subregistro.

Se han observado serias deficiencias en la información antes citada, sobre todo en las estructuras por edad censadas y, dado que la estimación de la cobertura de registro se basa en dichas estructuras, los sesgos obtenidos impiden, de manera directa (empleando el método de Preston), cuantificar la cobertura.

Después de diagnosticar algunas distorsiones en la información, se pasa a corregirlas, obteniéndose estimaciones más consistentes con la realidad mexicana. Lo anterior no debe interpretarse como que la estimación del grado de subregistro de la mortalidad adulta en México se ha evaluado con precisión, ya que, como se muestra en el presente trabajo, las distorsiones en la información no necesariamente se deben a los efectos señalados; no obstante, permiten el diagnóstico y evaluación de la información aquí planteados, y acercarse al conocimiento en materia de mortalidad adulta en México.

La importancia de conocer el grado de cobertura de las defunciones adultas en cualquier población humana radica en los efectos que tiene el fenómeno mortalidad frente a los demás fenómenos demográficos, y además, en la toma de decisiones que se basan en dicho fenómeno, por ejemplo, en políticas de población, programas de desarrollo urbano, programas de salud, etc. En resumen, el trabajo aquí planteado pretende servir a todo interesado en los niveles, tendencias, diferenciales y determinantes de la mortalidad en México para acercarlo al conocimiento de las dificultades con que se enfrenta la cuantificación de dicho fenómeno. Sin duda, en el futuro se desarrollarán y mejorarán las técnicas aquí expuestas, que facilitarán la estimación de la cobertura de las muertes adultas y con ello podrá corregirse el grado de subregistro de las mismas, y así estar en posibilidades de explicar con precisión el efecto de los niveles de la mortalidad en México.

* Las poblaciones estables se obtuvieron empleando el programa INTSP. Ver: US. Department of Commerce; "Computer Programs for Demographic Analysis", Bureau of the Census, junio 1976. 


\section{Apéndice 1}

MÉxico: Tablas de vida obtenidas a partir de las estructuras por edad de la población, defunciones y tasas de mortalidad, por sexo, 1940-1980.

$$
\text { Cuadro } 6
$$

MÉxICO: TABLAS DE VDA OBTENTDAS A PARTTR DE LAS ESTRUCTURAS FOR EDAD DE LA POBLACTÓN, DEFUNCTONES Y TASAS DE MORTALIDAD, POR SEXO, 1940-1980

\begin{tabular}{|c|c|c|c|c|c|c|c|c|c|c|c|c|c|}
\hline \multicolumn{14}{|c|}{ MORTALIDAD, POR SEXO, 1940-1980 } \\
\hline \multirow[b]{2}{*}{$\mathbf{x}$} & \multicolumn{7}{|c|}{ Hombses } & \multirow[b]{2}{*}{$\begin{array}{c}1960 \\
\underset{8 \pi}{D}\end{array}$} & \multirow[b]{2}{*}{$l_{x x}^{M}$} & \multirow[b]{2}{*}{$e^{p}$} & \multirow[b]{2}{*}{$\begin{array}{c}1970 \\
\mathbb{D}\end{array}$} & \multirow[b]{2}{*}{$e_{x}^{M}$} & \multirow[b]{2}{*}{$\begin{array}{c}1980 \\
e^{p}\end{array}$} \\
\hline & $e_{x}^{p}$ & $\begin{array}{c}1940 \\
\quad D \\
\& \times\end{array}$ & $\frac{M}{2 x}$ & $e_{x}^{p}$ & $\begin{array}{c}1950 \\
D \\
2 \pi\end{array}$ & $e^{M}$ & $e^{p}$ & & & & & & \\
\hline & & & 0 & & 1. & 0 & 1.00000 & 30 & $\infty$ & & & 10 & \\
\hline 15 & & & & & & & & & & & & & \\
\hline 20 & 0. & & & & & & & & & & & & 99126 \\
\hline 2 & & & & & 08 & & & & & & & & 0330 \\
\hline 30 & 0.79 & & 0.85901 & 0.8 & & & & & & & & 0.94943 & 0.73433 \\
\hline 35 & 0.84 & 0.83531 & 0.80 & 0.84 & & & & & & & & & 0.71045 \\
\hline 40 & 0.79 & 0.72 & 0.74 & & & 0.82294 & & & & & & & 0.70921 \\
\hline 45 & 0.65 & 0.71601 & & 0.86 & & & & & & & & 0.86304 & 0.67906 \\
\hline 50 & 0.57 & & 0.6 & 0.82 & & & & & & & & & \\
\hline 55 & 0.48687 & 0.59 & & & & & & & & & & & 0.58552 \\
\hline 50 & 0.459 & $0.5290 \mathrm{~J}$ & 0. & & 0. & 0. & 0.7 & & & & & & 0.52341 \\
\hline 65 & 0.38 & & & & & & & & & & & & \\
\hline 70 & 0.26 & 0.35 & & & 0. & 0. & & & & & & & \\
\hline 75 & 0.18830 & 0.26045 & 0.18 & 0.30 & & & & & & & & & \\
\hline 80 & 0.12 & 0.18574 & 0.10 & 0.21 & 0.2 & & & & & & & & \\
\hline 85 & 0.0952 & 0.10911 & 0.04799 & 0.16725 & 472 & 0.09674 & 0.27114 & 0.18865 & 0.16053 & 0.25058 & 0.20538 & 0.16844 & \\
\hline
\end{tabular}

Mujeros

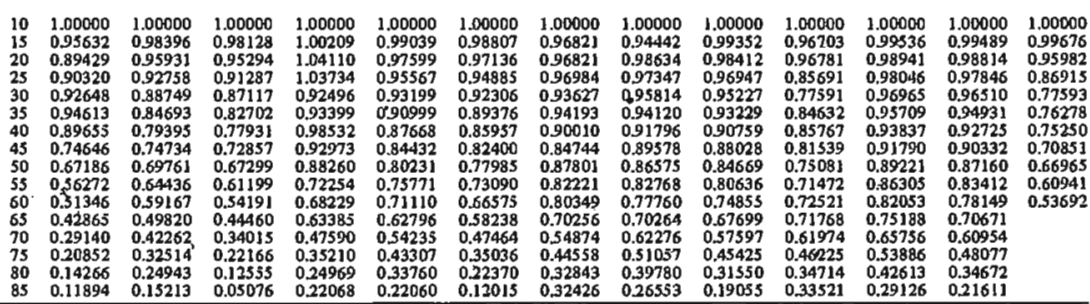

\section{Gráfica 9}

$$
P \quad D \quad M
$$

MÉxico: DisTribuciones DE LOS VALORES $\ell X$, lX y lX, POR SEXO, 1940-1980 H O M B $B$ B E $5^{-}$
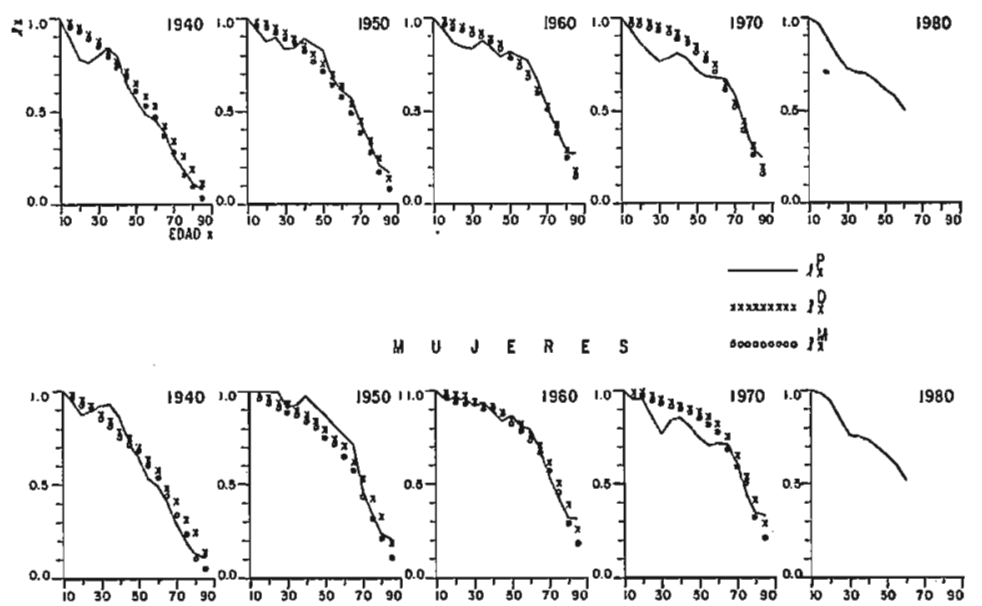
Apéndice 2

MÉx ICO: Gráficas y cuadros de los valores ${ }_{5} \hat{N}_{a} /{ }_{5} N_{a}, \hat{N}(a+) / N(a+), N(x) / N(a+)$ \& $D(x+) / N(x+)$, por sexo, 1940-1970.

\begin{tabular}{|c|c|c|c|c|c|c|c|c|}
\hline \multicolumn{9}{|c|}{ Cuadro 7} \\
\hline \multicolumn{9}{|c|}{ 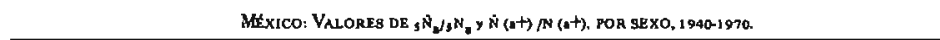 } \\
\hline \multirow{2}{*}{ Edad } & \multicolumn{8}{|c|}{ Hombres } \\
\hline & ${ }_{8} \mathrm{Na}_{5} / \mathrm{N}_{1}$ & 0 & ${ }_{5} \mathrm{~N}_{2} / 5 \mathrm{~N}^{19}$ & $n(3+) / N(a+)$ & ${ }_{3}, \mathrm{~N}_{\mathrm{a}} / \mathrm{s} \mathrm{N}_{\mathrm{a}}^{19}$ & ${ }^{60} N\left(a_{+}\right) / N(a+)$ & ${ }_{3} \mathrm{~N}_{\mathrm{a}} / \mathrm{s}, \mathrm{N}$ & $N(a+) / N(a)$ \\
\hline & & & 1.199 & 1.228 & 1.097 & 1.146 & 1.098 & 1.224 \\
\hline $\begin{array}{l}15 \\
20\end{array}$ & 1.213 & 1.200 & 1.315 & 1.234 & 1.191 & 1.157 & 1.214 & 1.256 \\
\hline 25 & & & & & $\begin{array}{l}1.221 \\
1.221\end{array}$ & $\begin{array}{l}1.150 \\
1.30\end{array}$ & $\begin{array}{l}1.344 \\
1.347\end{array}$ & $\begin{array}{l}1.267 \\
1.256\end{array}$ \\
\hline 30 & 1.182 & 1.160 & 1.423 & 1.196 & 108 & & 1377 & 1.234 \\
\hline 35 & 0.938 & 1.155 & 1.110 & 1.146 & 1.053 & 1.084 & 1.188 & 1.201 \\
\hline 40 & 1.159 & 1.236 & 1.169 & 1.157 & 1.234 & 1.093 & 1.259 & 1.204 \\
\hline 45 & 1.188 & 1.261 & 1.047 & 1.153 & 1.116 & 1.055 & 1.190 & 1.188 \\
\hline & & & ${ }_{1.375}^{1.14}$ & & $\begin{array}{l}1.042 \\
1072\end{array}$ & & & 1.188 \\
\hline 60 & 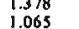 & $\frac{1.311}{1.282}$ & $\begin{array}{l}1.375 \\
1.047\end{array}$ & .1 .177 & ${ }_{0893}^{1072}-x-3$ & 1.033 & 1.273 & .1 .133 \\
\hline 65 & 1.438 & 1.439 & 1.238 & 1.261 & 1.210 & 1.095 & 1.055 & 1.071 \\
\hline 70 & & 1.440 & 1.250 & 1.276 & 1.075 & 1.032 & 1.032 & 1.83 \\
\hline 75 & 1.669 & 1.455 & 1.474 & 1.299 & 1.233 & 1.000 & 1.346 & 0.128 \\
\hline \multirow[t]{2}{*}{$\begin{array}{l}80 \\
85\end{array}$} & $\begin{array}{l}1.1505 \\
0.927\end{array}$ & $\begin{array}{l}1.266 \\
0.927\end{array}$ & $\begin{array}{l}1.353 \\
0.863\end{array}$ & $\begin{array}{l}1.144 \\
0.863\end{array}$ & $\begin{array}{l}1.1 .151 \\
0.522\end{array}$ & $\begin{array}{l}0.884 \\
0.522\end{array}$ & $\begin{array}{l}1.204 \\
0.675\end{array}$ & $\begin{array}{l}0.966 \\
0.675\end{array}$ \\
\hline & \multirow{2}{*}{\multicolumn{8}{|c|}{ Mujeres }} \\
\hline 10 & & & & & & & & \\
\hline & & & 1200 & 120 & 104 & 1.106 & 1170 & 1.2378 \\
\hline 20 & & 1.176 & 0.1151 & 1.201 & 0.154 & 1.163 & 1.203 & 1.252 \\
\hline & 1.072 & 1.154 & 1.159 & 1.213 & 1.143 & i. 165 & 1.260 & 1.264 \\
\hline 30 & 1.126 & 1.174 & 1.391 & 1.227 & 1.204 & 1.177 & 1.358 & 1.263 \\
\hline $\begin{array}{l}35 \\
40\end{array}$ & & & 1.075 & 1.191 & 1.090 & $\begin{array}{l}1.163 \\
\end{array}$ & 1.163 & 1.243 \\
\hline & 1.168 & 1.280 & $\begin{array}{l}1.138 \\
1.188\end{array}$ & 1250 & 1.159 & 1.168 & 1.268 & $\begin{array}{l}1.267 \\
1.267\end{array}$ \\
\hline 50 & 1213 & & $11106^{\circ}$ & 30 & 105 & 106 & 1,304 & $\begin{array}{l}1.28 \\
\end{array}$ \\
\hline & 1.445 & 1.467 & 1.506 & 1.363 & 1.215 & 1.185 & $\begin{array}{l}1.339 \\
1.339\end{array}$ & 1.228 \\
\hline & & 1.476 & 1.104 & 1.312 & 1.014 & 1.173 & 1.162 & 1.187 \\
\hline 65 & $\begin{array}{l}1.050 \\
0\end{array}$ & 1.6933 & $\begin{array}{l}1.427 \\
\end{array}$ & 1.445 & 1.3727 & $\begin{array}{r}1.229 \\
129\end{array}$ & 1.158 & 1.199 \\
\hline & $\begin{array}{l}1.652 \\
2.38\end{array}$ & 1.720 & 1.386 & 1.456 & $\begin{array}{l}1.231 \\
.507\end{array}$ & 1.2177 & $\begin{array}{l}1.216 \\
1547\end{array}$ & 1.223 \\
\hline \multirow{3}{*}{85} & 1.808 & 1.996 & 1.500 & 1.273 & 1.303 & $\begin{array}{l}1.2000 \\
0.998\end{array}$ & 1.312 & $\begin{array}{l}1.017 \\
1.017\end{array}$ \\
\hline & 1.079 & 1.079 & 0.971 & $0.97 i$ & 0.684 & 0.684 & 0.708 & 0.708 \\
\hline & \multicolumn{8}{|c|}{ Gráfica 10} \\
\hline
\end{tabular}

Míxico: Distribución DE LOS VALORES ${ }_{5} \hat{\mathrm{N}}_{\mathbf{X}} / \mathrm{N}_{\mathbf{X}}$, HOMBRES, $1940-1970$

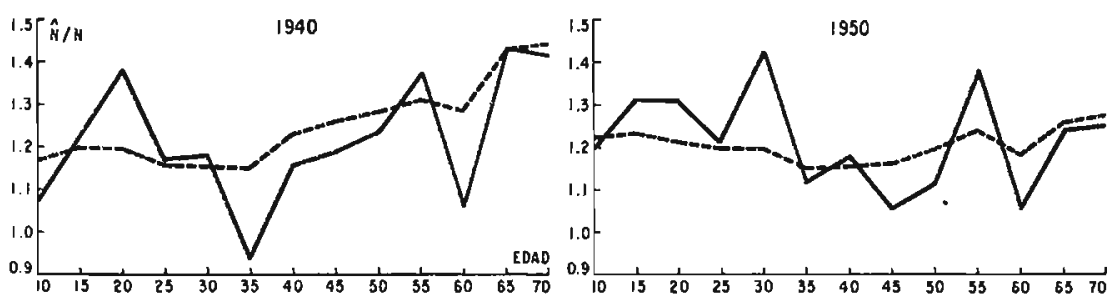

- ${ }^{3} \hat{N}_{x} / 5^{N_{x}}$

$-\cdots(x+1 / N(x+)$

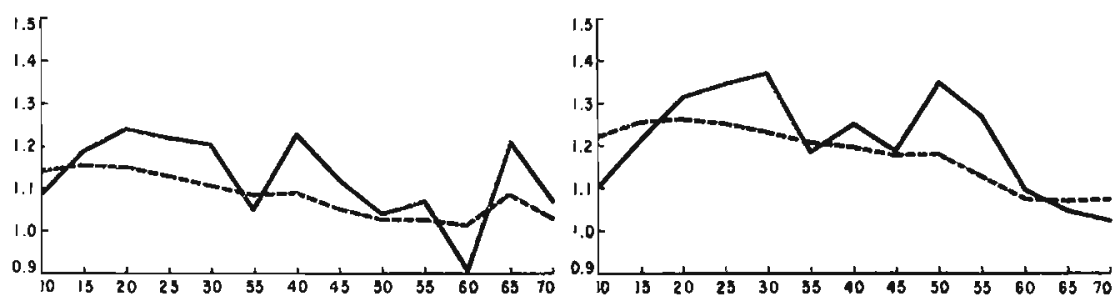




\section{Gráfica 11}

MÉXICO: DistribuCión DE VALORES ${ }_{5} \hat{\mathrm{N}}_{\mathrm{X}} / \mathrm{N}_{\mathrm{x}} \cdot \mathrm{Y} \hat{\mathrm{N}}(\mathrm{x}+\mathrm{x} / \mathrm{N}(\mathrm{x}+)$, MUJERES, 1940-1970

1940

1950

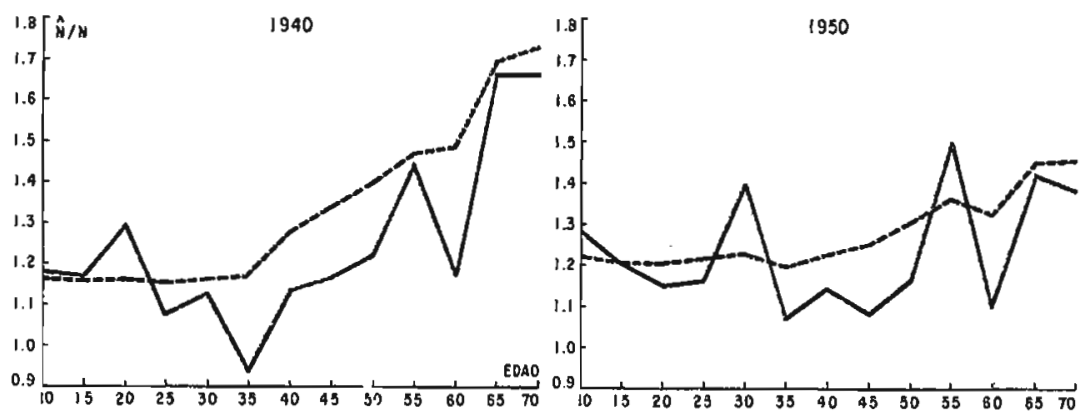

$-{ }_{3} \hat{N}^{5} /{ }^{3} N^{2}$

$\hat{N}(x+1) / N(x+1)$

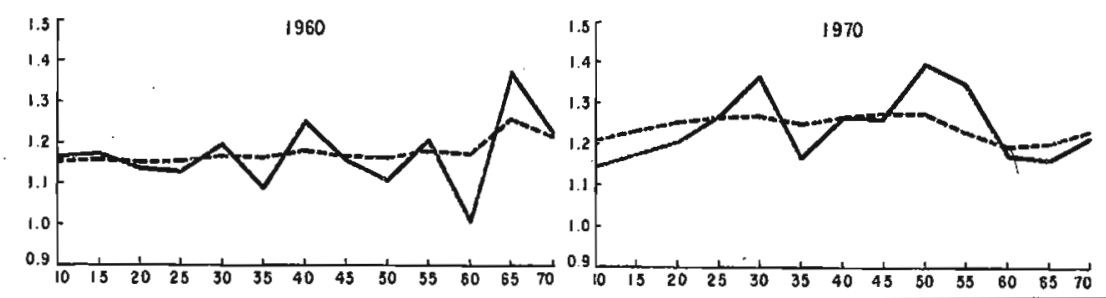

\section{Cuadro 8}

MÉXICO: VALORES DE N(x)/N( $\left.x^{+}\right)$y $D\left(x^{+}\right) / N\left(x^{+}\right)$, POR SEXO, 1940-1970.

\begin{tabular}{|c|c|c|c|c|c|c|c|c|c|}
\hline & \multicolumn{8}{|c|}{ Hombres } & $(x+2) N(x+)$ \\
\hline & $\begin{array}{l}10 \\
15 \\
20 \\
25 \\
30 \\
35 \\
40 \\
40 \\
45 \\
30 \\
35 \\
60 \\
65 \\
70 \\
73 \\
73 \\
80 \\
85\end{array}$ & $\begin{array}{l}.040 \\
.040 \\
.097 \\
.099 \\
.045 \\
.053 \\
.063 \\
.063 \\
.065 \\
.071 \\
.084 \\
.110 \\
.110 \\
.131 \\
.147 \\
.242\end{array}$ & $\begin{array}{l}.016 \\
.019 \\
.021 \\
.024 \\
.027 \\
.031 \\
.036 \\
.043 \\
.031 \\
.052 \\
.053 \\
.099 \\
.125 \\
.139 \\
.203 \\
.275\end{array}$ & 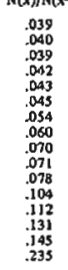 & 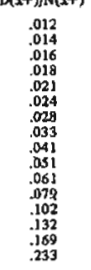 & $\begin{array}{l}.042 \\
.042 \\
.042 \\
.094 \\
.041 \\
.043 \\
.048 \\
.062 \\
.052 \\
.060 \\
.069 \\
.062 \\
.062 \\
.000 \\
.098 \\
.119 \\
.123 \\
.192\end{array}$ & 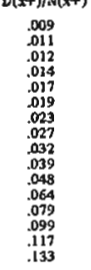 & 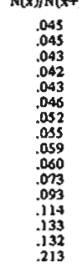 & 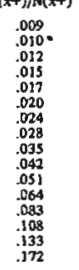 \\
\hline & $\begin{array}{l}100 \\
13 \\
20 \\
23 \\
30 \\
35 \\
40 \\
45 \\
45 \\
50 \\
55 \\
60 \\
65 \\
70 \\
75 \\
80 \\
85\end{array}$ & 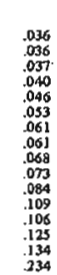 & 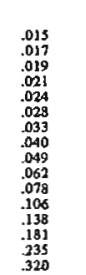 & $\begin{array}{l}.036 \\
.037 \\
.041 \\
.044 \\
.043 \\
.045 \\
.055 \\
.059 \\
.067 \\
.068 \\
.075 \\
.0701 \\
.305 \\
.124 \\
.130 \\
.232\end{array}$ & 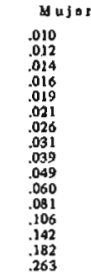 & $\begin{array}{l}0 . \\
.039 \\
.090 \\
.091 \\
.094 \\
.045 \\
.045 \\
.043 \\
.051 \\
.051 \\
.060 \\
.067 \\
.007 \\
.004 \\
.094 \\
.114 \\
.120 \\
203\end{array}$ & 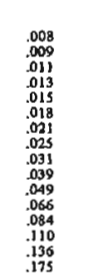 & $\begin{array}{l}.092 \\
.043 \\
.044 \\
.044 \\
.044 \\
.046 \\
.052 \\
.054 \\
.056 \\
.058 \\
.070 \\
.098 \\
.105 \\
.115 \\
.119 \\
.205\end{array}$ & $\begin{array}{l}.007 \\
.009 \\
.010 \\
.012 \\
.012 \\
.017 \\
.021 \\
.026 \\
.032 \\
.039 \\
.048 \\
.063 \\
.083 \\
.109 \\
.136 \\
1.12\end{array}$ \\
\hline
\end{tabular}




\section{Gráfica 12}

MÉXICO: GRÁFICAS DE D(a+)/N(a+) CONTRA N(a)/N(a+), POR SEXO, 1940-1970

H O M B R E

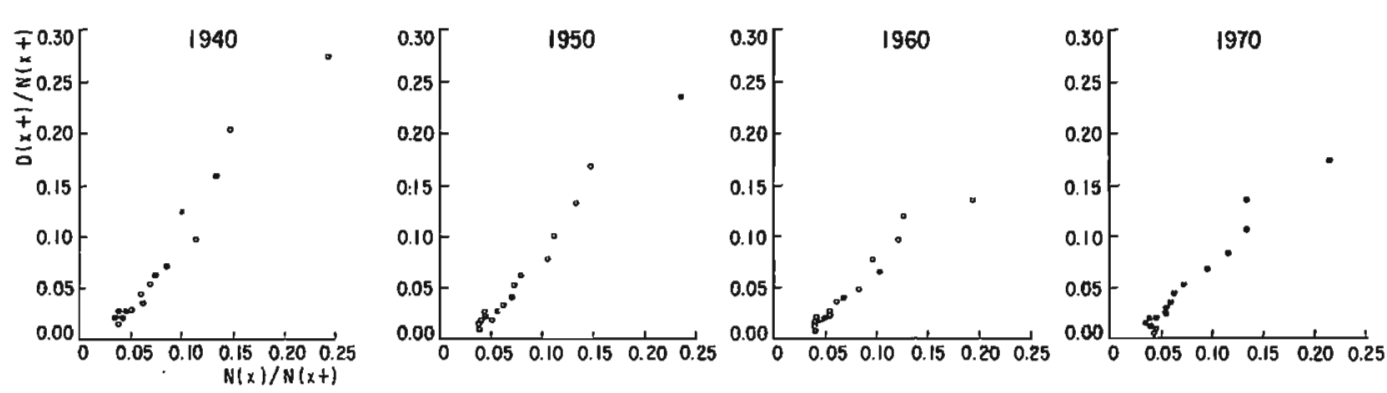

M U J E R E S
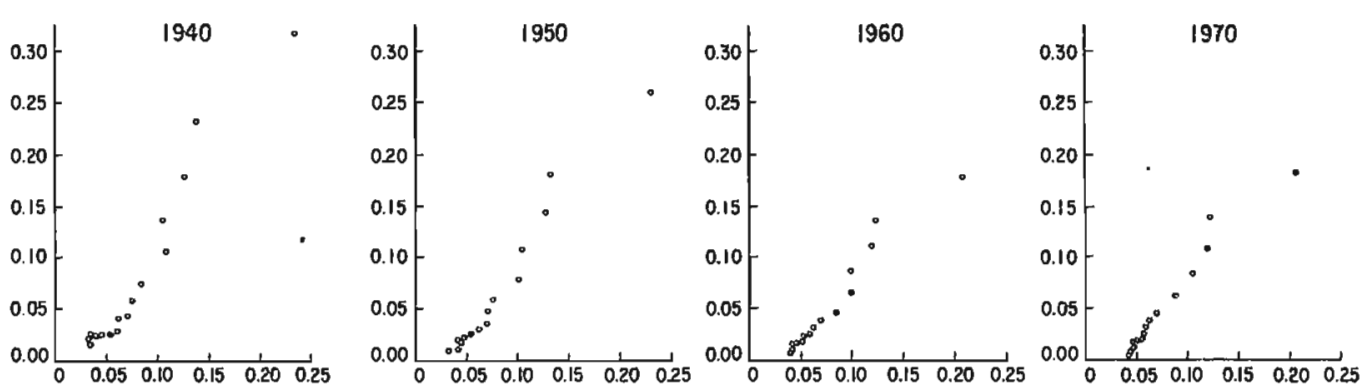


\section{Apéndice 3}

Mé XICO: Gráficas y cuadros de los valores $\hat{N}[(x+5)+] / N(x+)$, por sexo, 1930-1970. y ${ }_{10} \hat{N}_{a} /{ }_{10} N_{a}-5$, hombres, 1930.

\section{Gráfica 13}

MÉXICO: DisTRIBUCión DE LOS VALORES N̂ $((x+5)+) / N(x+)$, POR SEXO, 1930

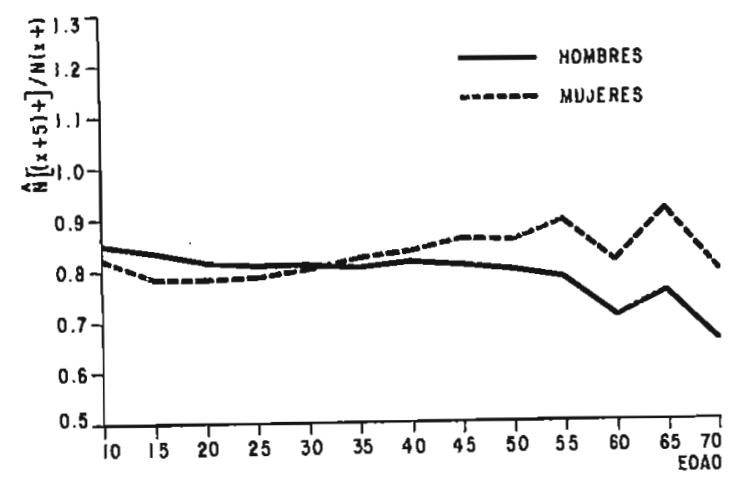

\section{Gráfica 14}

MÉXICO: DisTRIBUCIÓN DE LOS VALORES $\hat{\mathrm{N}}((\mathrm{x}+5)+) / \mathrm{N}(\mathrm{x}+)$, POR SEXO, 1940-1970
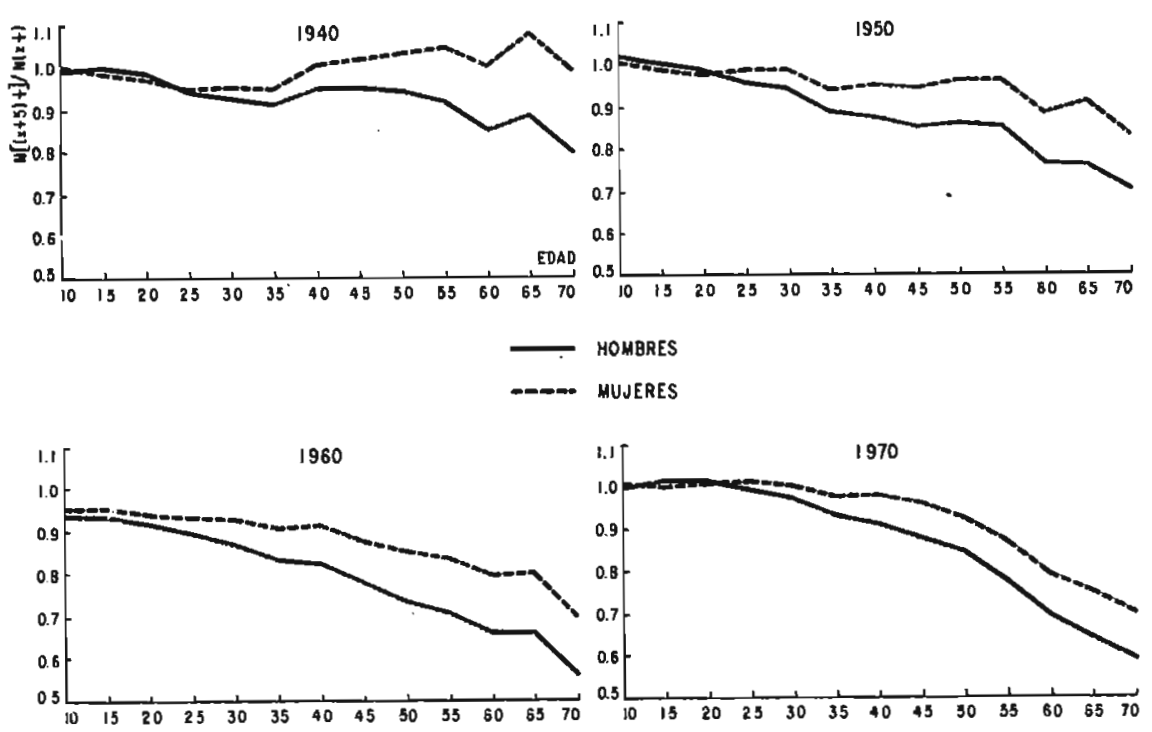
Cuadro 9

MÉXICO: VALORES DE $\hat{\mathrm{N}}((\mathrm{x}+5)+) / \mathrm{N}(\mathrm{x}+)$ POR SEXO, $1930-1970$

\begin{tabular}{|c|c|c|c|c|c|}
\hline $\mathbf{x}$ & 1930 & 1940 & $\begin{array}{l}A \text { ño } \\
1950\end{array}$ & 1960 & 1970 \\
\hline \multicolumn{6}{|c|}{ Hom bres } \\
\hline 10 & .858 & .980 & 1.010 & .937 & .999 \\
\hline 15 & .841 & .989 & 1.006 & .939 & 1.019 \\
\hline 20 & .824 & .975 & .983 & .924 & 1.019 \\
\hline 25 & .814 & .933 & .956 & .899 & 1.000 \\
\hline 30 & .819 & .918 & .941 & .871 & .971 \\
\hline 35 & 810 & 899 & .888 & .839 & .930 \\
\hline 40 & .821 & .944 & .879 & .830 & .916 \\
\hline 45 & .813 & .940 & .855 & .782 & .883 \\
\hline 50 & .803 & .930 & .861 & .743 & .855 \\
\hline 55 & .789 & .902 & .850 & .712 & .781 \\
\hline 60 & .707 & .836 & .768 & .666 & .703 \\
\hline 65 & .756 & .875 & .767 & .668 & .647 \\
\hline 70 & .656 & .789 & .704 & .568 & .597 \\
\hline 75 & .553 & .671 & .610 & .469 & .535 \\
\hline 80 & .292 & .384 & .368 & .272 & .317 \\
\hline
\end{tabular}

Mujeres

\begin{tabular}{lrrrrr}
10 & .829 & .985 & 1.006 & .959 & 1.000 \\
15 & .795 & .975 & .987 & .952 & 1.009 \\
20 & .788 & .966 & .979 & .943 & 1.015 \\
25 & .795 & .937 & .980 & .937 & 1.016 \\
30 & .818 & .942 & .980 & .931 & 1.006 \\
35 & .821 & .939 & .938 & .913 & .976 \\
40 & .844 & .995 & .950 & .915 & .979 \\
45 & .866 & 1.011 & .947 & .878 & .959 \\
50 & .864 & 1.026 & .961 & .855 & .933 \\
55 & .895 & 1.036 & .965 & .839 & .870 \\
60 & .813 & .990 & .883 & .792 & .799 \\
65 & .918 & 1.065 & .910 & .802 & .756 \\
70 & .794 & 978 & .834 & .697 & .704 \\
75 & .699 & .847 & .736 & .589 & .606 \\
80 & .348 & .461 & .418 & .337 & .346 \\
\hline
\end{tabular}




\section{Gráfica 15}

MÉXICO: Distribución DE LOS VALORES ${ }_{10} \hat{\mathrm{N}}_{\mathrm{a}-5} /{ }_{10} \mathrm{~N}_{\mathrm{a}-5} \mathrm{Y}_{10} \hat{\mathrm{N}}_{\mathrm{a}} /{ }_{10} \mathrm{~N}_{\mathrm{a}-5}$, HOMBRES $1930-1935$

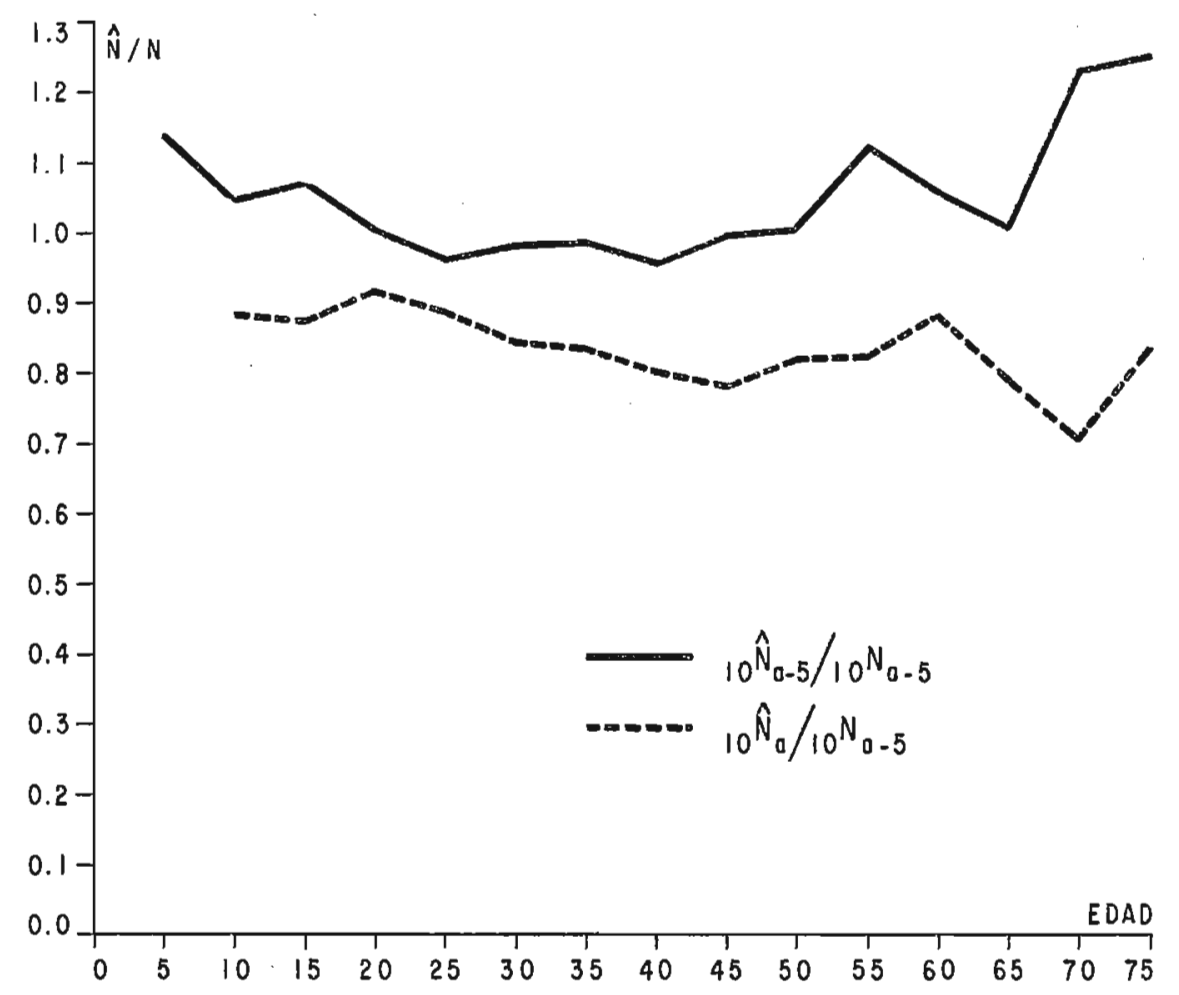




\section{Apéndice 4}

México: Gráfica y cuadro de los valores $\hat{N}[(x+5)+] / N(x+)$, por sexo,

1930- 1970.

\section{Cuadro 10}

MÉXICO: VALORES DE N $(X+5)+$ ) / $\mathrm{N}(\mathrm{X}+$ ) POR SEXO. 1930-1970

\begin{tabular}{|c|c|c|c|c|c|}
\hline \multicolumn{6}{|c|}{ Año } \\
\hline$x+5$ & 1930 & 1940 & 1950 & 1960 & 1970 \\
\hline \multicolumn{6}{|c|}{ Hombres } \\
\hline 10 & 0.8470 & 0.9906 & 1.0341 & 0.9395 & 0.9930 \\
\hline 15 & 0.8387 & 0.9864 & 1.0327 & 0.9379 & 0.9924 \\
\hline 20 & 0.8279 & 0.9808 & 1.0301 & 0.9355 & 0.9910 \\
\hline 25 & 0.8166 & 0.9746 & 1.0274 & 0.9325 & 0.9892 \\
\hline 30 & 0.8058 & 0.9674 & 1.0239 & 0.9287 & 0.9868 \\
\hline 35 & 0.7949 & 0.9597 & 1.0193 & 0.9237 & 0.9826 \\
\hline 40 & 0.7853 & 0.9536 & 1.0140 & 0.9182 & 0.9776 \\
\hline 45 & 0.7776 & 0.9509 & 1.0089 & 0.9123 & 0.9732 \\
\hline 50 & 0.7716 & 0.9510 & 1.0056 & 0.9060 & 0.9673 \\
\hline 5.5 & 0.7689 & 0.9542 & 1.0055 & 0.9002 & 0.9617 \\
\hline 60 & 0.7660 & 0.9585 & 1.0064 & 0.8948 & 0.9545 \\
\hline 65 & 0.7697 & 0.9723 & 1.0123 & 0.8963 & 0.9457 \\
\hline 70 & 0.7923 & 1.0039 & 1.0289 & 0.8984 & 0.9352 \\
\hline 75 & 0.8367 & 1.0526 & 1.0620 & 0.9104 & 0.9407 \\
\hline 80 & 0.8889 & 1.1207 & 1.1034 & 0.9194 & 0.9524 \\
\hline 85 & 0.8000 & 1.0526 & 1.0500 & 0.8636 & 0.8696 \\
\hline \multicolumn{6}{|c|}{ Mujeres } \\
\hline 10 & 0.8408 & 1.0030 & 1.0469 & 0.9763 & 1.0073 \\
\hline 15 & 0.8351 & 1.0026 & 1.0516 & 0.9797 & $1.01 ! 7$ \\
\hline 20 & 0.8274 & 1.0002 & 1.0558 & 0.9828 & 1.0158 \\
\hline 25 & 0.8198 & 0.9969 & 1.0603 & 0.9860 & 1.0203 \\
\hline 30 & 0.8128 & 0.9927 & 1.0650 & 0.9890 & 1.0245 \\
\hline 35 & 0.8064 & 0.9868 & 1.0693 & 0.9915 & 1.0280 \\
\hline 40 & 0.8005 & 0.9799 & 1.0728 & 0.9941 & 1.0316 \\
\hline 45 & 0.7958 & 0.9719 & 1.0767 & 0.9959 & 1.0345 \\
\hline 50 & 0.7913 & 0.9608 & 1.0803 & 0.9961 & 1.0370 \\
\hline 55 & 0.7882 & 0.9452 & 1.0869 & 0.9968 & 1.0374 \\
\hline 60 & 0.7856 & 0.9197 & 1.0925 & 0.9970 & 1.0362 \\
\hline 65 & 0.7934 & 0.8834 & 1.1013 & 1.0000 & 1.0358 \\
\hline 70 & 0.8201 & 0.8286 & 1.1250 & 1.0107 & 1.0391 \\
\hline 75 & 0.8686 & 0.7216 & 1.1677 & i. 0253 & 1.5000 \\
\hline 80 & 0.9231 & 1.2394 & 1.2055 & 1.0395 & 1.0513 \\
\hline 85 & 0.8462 & 1.1250 & 1.1154 & 0.9310 & 0.9333 \\
\hline
\end{tabular}




\section{Gráfica 16}

MÉXICO: Distribución DE los VALORES $\hat{N}(X+5)+) / N(X+)$, POR SEXO, 1930-1970

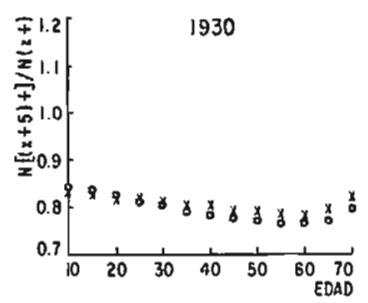

00000 - HOHBRES

XXYXX MUJERES
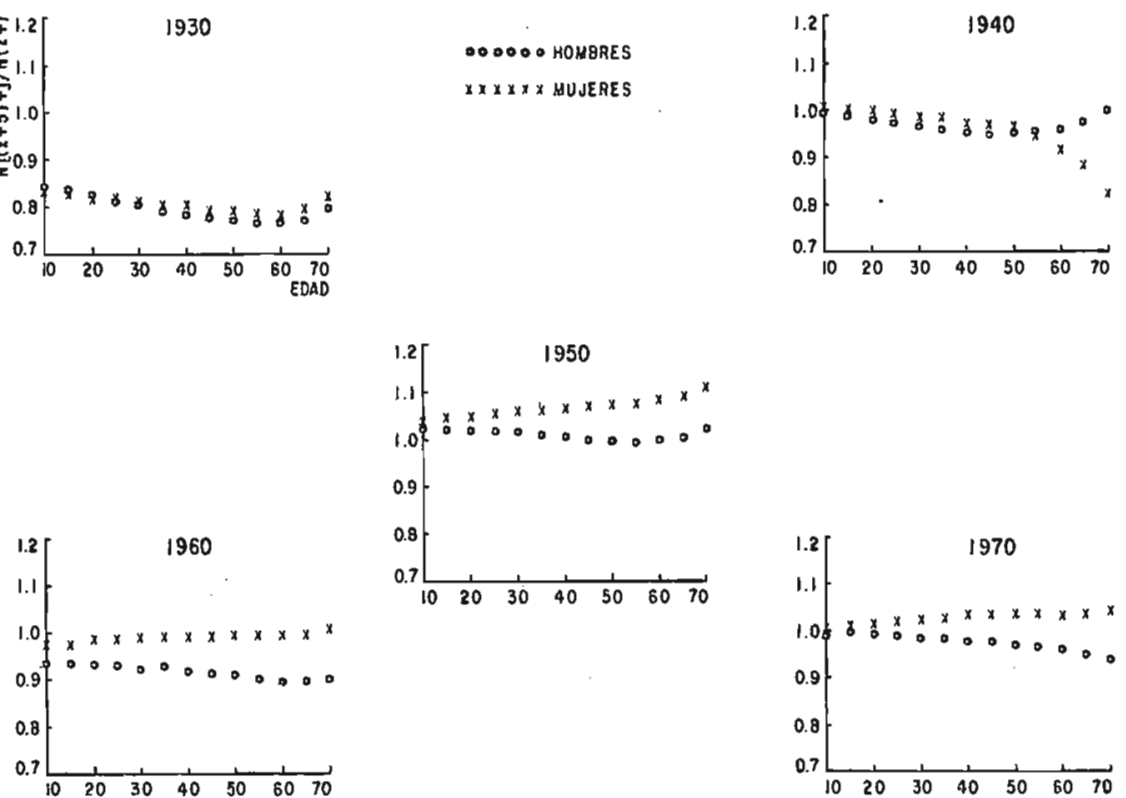

\section{Referencias}

Barclay, George W.; Coale, Ansley J.; Stoto, Michael A.; and Trussell, T. James. 1976. A reassessment of the demography of traditional rural China. Population Index (Princeton, N.J.) 42 (4): 606-635.

BENNETT, G. Neil and Horiuchi, Shiro; 1981. "Estimating the Completeness of Death Registration in a Closed Population"'.Population Index (Princeton, N.J.), 47 (2): 207.222 .

Brass, William; and Coale, Ansley J. 1968. Methods of analysis and estimation. In The Demography of Tropical Africa, by William Brass et al., pp. 88-139. Princeton, N.J.: Princeton University Press.

-1 , 1975. Methods for Estimating Fertility and Mortality from Limited and Defective Data: Based on Seminars Held 16-24 September 1971 at the Centro Latinoamericano de Demografía (CELADE) San José, Costa Rica. Chapel Hill, N.C.: University of North Carolina, International Program of Laboratories for Population Statistics xii, $159 \mathrm{pp}$.

, 1979. A procedure for comparing mortality measures calculated from 
intercensal survival with the corresponding estimates from registered deaths. Asian and Pacific Census Forum (Honolulu) 6 (2): 5-7.

CoAle, Ansley J.; and Demeny, Paul. 1966. Regional Model Life Tables and Stable Populations. Princeton, N.J.: Princeton University Press. xiii, $871 \mathrm{pp}$.

COALE, Ansley J. 1972. The Growth and Structure of Human Populations: A for Princeton University, Office of Population Research. xii, $227 \mathrm{pp}$.

, Cho, Lee-Jay; and Goldman, Noreen. 1980 Estimation of Recent Trends in Fertility and Mortality in the Republic of Korea. Report No. 1. Washington: National Academy of Sciences for National Research Council, Committee on Population and Demography. xiv, $77 \mathrm{pp}$.

KDREA, Republic. Economic Planning Board. National Bureau of Statistics. 1972. 1970 Population and Housing Census Report. Vol. 1. Complete Enumeration. No. 12-1. Republic of Korea. Seoul: Economic Planning Board, National Bureau of Statistics. 416 pp.

- Economic Planning Board. National Bureau of Statistics. 1977. 1975 Population and Housing Census Report. Vol. 1. Complete Enumeration. No. 12-1. Whole Country. Seoul: Economic Planning Board, National Bureau of Statistics. $368 \mathrm{pp}$.

MARTIN, Linda. 1977. Measuring Completeness of Death Registration in Destabilized Populations. Ph. D. thesis, Princeton University.

$\longrightarrow, 1980$. A modification for use in destabilized populations of Brass's technique for estimating completeness of death registration. Population Studies (London) 34 (2): 381-395.

Preston, Samuel H.; and Palloni, Alberto. 1977. Fine-tuning Brass-type mortality estimates with data on ages of surviving children. Population Bulletin of the United Nations (New York), No. 10. Pp. 72-91.

PrEston, Samuel; and Hill, Kenneth. 1980. Estimating the completeness of death registration. Population Studies (London) 34 (2): 349-366.

Preston, Samuel; Coale, Ansley J.; Trussell, James; and Weinstein, Maxime. 1980 Estimating the completeness of reporting of adult deaths in populations that are aproximately stable. Population Index (Princeton, N.J.) 46 (2): 179-202.

Sullivan, Jeremiah M. 1972. Models for the estimation of the probability of dying between birth and exact ages of early childhood. Population Studies (London) 29 (1): $79: 97$.

SwEDEN. Statistiska Centralbyrăn. 1966-1967. Folkmangdens Forandringar Population Changes. 1965 and 1966 editions Stockholm: Statistiska Centralbyrån.

- Statistiska Centralbyrån. 1969-1972. Befolkningsförändringar. . . Del 3. Hela Riket och Länen $m m$ Population Changes. . Part 3. The Whole Country and the Counties etc. 1967, 1968, and 1970 editions. Stockholm: Stastiska Centralbyrån.

- Statistiska Centralbyrån. 1974. Livslangdstabeller for Artiondet 19611970 Life Tables for the Decade 1961-1970 Stockholm: Statistiska Centralbyrån.

TRUSSELL, T. James. 1975. A re-estimation of the multiplying factors for the 
Brass technique for determining childhood survivorship rates. Population Studies (London) 29 (1): 97-107.

, and Manken, Jane. 1969. Estimating the completeness of deaths and relative underenumeration in two successive censuses. Asian and Pacific Census Forum (Honolulu) 6 (2): 9-11.

U.N. DEPARTMENT of International Economic and Social Affairs. Population Division. 1979. Model Life Tables for Developing Countries: An Interim Report. Working Paper, No. 63. ESA/P/WP. 63. New York: U.N., Department of International Economic and Social Affairs. $89 \mathrm{pp}$.

Vincent, Paul. 1951. La mortalité des vieillards The mortality of the aged Population (Paris) 6 (2): 182-204. 